\title{
Estimating the number of Reeb chords using a linear representation of the characteristic algebra
}

\author{
Georgios Dimitroglou Rizell \\ ROMAN GOLOVKO
}

\begin{abstract}
Given a chord-generic, horizontally displaceable Legendrian submanifold $\Lambda \subset P \times \mathbb{R}$ with the property that its characteristic algebra admits a finite-dimensional matrix representation, we prove an Arnold-type lower bound for the number of Reeb chords on $\Lambda$. This result is a generalization of the results of Ekholm, Etnyre, Sabloff and Sullivan, which hold for Legendrian submanifolds whose Chekanov-Eliashberg algebras admit augmentations. We also provide examples of Legendrian submanifolds $\Lambda$ of $\mathbb{C}^{n} \times \mathbb{R}, n \geq 1$, whose characteristic algebras admit finite-dimensional matrix representations but whose Chekanov-Eliashberg algebras do not admit augmentations. In addition, to show the limits of the method of proof for the bound, we construct a Legendrian submanifold $\Lambda \subset \mathbb{C}^{n} \times \mathbb{R}$ with the property that the characteristic algebra of $\Lambda$ does not satisfy the rank property. Finally, in the case when a Legendrian submanifold $\Lambda$ has a non-acyclic Chekanov-Eliashberg algebra, using rather elementary algebraic techniques we obtain lower bounds for the number of Reeb chords of $\Lambda$. These bounds are slightly better than the number of Reeb chords it is possible to achieve with a Legendrian submanifold whose Chekanov-Eliashberg algebra is acyclic.
\end{abstract}

53D12; 53D42

\section{Introduction}

\subsection{Geometric background}

The contactization of an exact symplectic $2 n$-manifold $(P, d \theta)$ is a contact manifold $P \times \mathbb{R}$ equipped with the contact structure $\xi:=\operatorname{ker}(d z+\theta)$, where $z$ is a coordinate on $\mathbb{R}$. Let $\Lambda$ be an $n$-dimensional submanifold of $P \times \mathbb{R}$. We say that $\Lambda$ is a Legendrian submanifold if and only if $T_{x} \Lambda \subset \xi_{x}$ for all $x \in \Lambda$. A smooth 1-parameter family of Legendrian submanifolds is called a Legendrian isotopy. We will always assume that $(P, d \theta)$ has finite geometry at infinity.

We note that if $\Lambda \subset P \times \mathbb{R}$ is a Legendrian submanifold, then double points of the socalled Lagrangian projection $\Pi_{L}: P \times \mathbb{R} \rightarrow P$ defined by $\Pi_{L}(x, z)=x$ correspond 
bijectively to integral curves of $\partial_{z}$ in $P \times \mathbb{R}$ having endpoints on $\Lambda$. The vector field $\partial_{z}$ is the Reeb field of the contact form $d z+\theta$ and its integral curves having endpoints on $\Lambda$ are called Reeb chords on $\Lambda$. The set of Reeb chords on $\Lambda$ will be denoted by $\mathcal{Q}(\Lambda)$. We say that $\Lambda$ is chord-generic if all self-intersections of the Lagrangian immersion $\Pi_{L}(\Lambda)$ are transverse double points, which, in the case when $\Lambda$ is closed, in particular implies that $|\mathcal{Q}(\Lambda)|<\infty$. Note that the chord-generic Legendrian embeddings of a closed manifold form an open and dense subset of the space of Legendrian embeddings.

From now on we assume that all Legendrian submanifolds are closed, orientable, connected and chord-generic, unless stated otherwise.

A Legendrian submanifold $\Lambda \subset P \times \mathbb{R}$ is called horizontally displaceable if the projection $\Pi_{L}(\Lambda)$ can be completely displaced off of itself by a Hamiltonian isotopy.

Observe that in the case $(P, d \theta)=\left(T^{*} M, d \theta_{M}\right)$, where $\theta_{M}$ is the so-called Liouville form, the bundle projection $p: T^{*} M \rightarrow M$ induces the natural projection $\Pi_{F}: T^{*} M \times$ $\mathbb{R} \rightarrow M \times \mathbb{R}$ defined by $\Pi_{F}(x, z)=(p(x), z)$. This projection is called the front projection. As a special case, this also applies to the standard symplectic vector space $\left(\mathbb{C}^{n}=T^{*} \mathbb{R}^{n},-d\left(y_{1} d x_{1}+\cdots+y_{n} d x_{n}\right)\right)$ (where we usually use minus the Liouville form).

There are two "classical" invariants for a Legendrian submanifold $\Lambda \subset \mathbb{C}^{n} \times \mathbb{R}$ which are invariant under Legendrian isotopy: the Thurston-Bennequin number $\operatorname{tb}(\Lambda)$ and the rotation class $r(\Lambda)$. The Thurston-Bennequin number of a homologically trivial Legendrian $\Lambda \subset P \times \mathbb{R}$ was first defined in the case $n=1$ by Bennequin [3], and independently by Thurston, and then extended to the case when $n \geq 1$ by Tabachnikov [30]. One may define it by $\operatorname{tb}(\Lambda):=\operatorname{lk}\left(\Lambda, \Lambda^{\prime}\right)$, where $\Lambda^{\prime}$ is a sufficiently small push-off of $\Lambda$ along the Reeb vector field. The rotation class $r(\Lambda)$ of a Legendrian submanifold $\Lambda \subset P \times \mathbb{R}$ is defined as the homotopy class of the complex bundle monomorphism $T \Lambda \otimes \mathbb{C} \rightarrow \xi \subset T P$ induced by the differential of the inclusion (together with some choice of compatible almost complex structure on $P$ ). We refer to Ekholm, Etnyre and Sullivan [8] for more details.

Legendrian contact homology is a modern invariant of Legendrian submanifolds in $P \times \mathbb{R}$. It is a variant of the symplectic field theory introduced by Eliashberg, Givental and Hofer [13]. Independently, for Legendrian knots in $\mathbb{C} \times \mathbb{R}$, it was defined by Chekanov [4]. This invariant associates a differential graded algebra (DGA) denoted by $\mathcal{A}(\Lambda)$ to a Legendrian submanifold $\Lambda$, sometimes called the Chekanov-Eliashberg algebra of $\Lambda . \mathcal{A}(\Lambda)$ is a non-commutative unital differential graded algebra over a field $\mathbb{F}$ freely generated by elements of $\mathcal{Q}(\Lambda)$. The differential $\partial(a)$ of a generator 
$a \in \mathcal{Q}(\Lambda)$ is given by a count of rigid pseudo-holomorphic disks for some choice of compatible almost complex structure, and is then extended using the Leibniz rule.

We will use the version of Legendrian contact homology for Legendrian submanifolds of $P \times \mathbb{R}$, which was developed by Ekholm, Etnyre and Sullivan [10]. It was shown there that the homology of $(\mathcal{A}(\Lambda), \partial)$, the so-called Legendrian contact homology of $\Lambda$, is independent of the choice of an almost complex structure and invariant under Legendrian isotopy.

We now sketch the definition of the differential, as given in [10]. For a generic tame almost complex structure $J$ on $P$, we define

$$
\partial(a)=\sum_{\operatorname{dim} \mathcal{M}_{a ; \boldsymbol{b} ; A}(\Lambda ; J)=0}(-1)^{(n-1)(|a|+1)} \#\left(\mathcal{M}_{a ; \boldsymbol{b} ; A}(\Lambda ; J)\right) \boldsymbol{b},
$$

where $\boldsymbol{b}=b_{1} \cdots b_{m}$ is a word of Reeb chords. Here $\mathcal{M}_{a ; \boldsymbol{b} ; A}(\Lambda ; J)$ is the modulispace of $J$-holomorphic disks in $P$ having boundary on $\Pi_{L}(\Lambda)$, a positive boundary puncture mapping to $a$, negative boundary punctures mapping to $b_{1}, \ldots, b_{m}$ (in that order relative to the oriented boundary with the positive puncture removed), and being in the relative homology class $A \in H_{2}\left(P, \Pi_{L}(\Lambda)\right)$. We refer to Section 3.3 for the definition of positive and negative punctures.

The Maslov class of a Legendrian submanifold $\Lambda$ is a cohomology class $\mu(\Lambda)$ in $H^{1}(\Lambda ; \mathbb{Z})$ that assigns to each 1 -dimensional homology class the Maslov index of a path representing that class; see eg [8]. In the case when the Maslov class vanishes, the Chekanov-Eliashberg algebra of a one-component Legendrian submanifold has a canonical grading in $\mathbb{Z}$. In general, the Chekanov-Eliashberg algebra of an oriented one-component Legendrian submanifold has a canonical grading in $\mathbb{Z}_{2}$.

Given a Chekanov-Eliashberg algebra $(\mathcal{A}(\Lambda), \partial)$ over a field $\mathbb{F}$, an augmentation of $(\mathcal{A}(\Lambda), \partial)$ is a unital algebra chain map $\varepsilon:(\mathcal{A}(\Lambda), \partial) \rightarrow(\mathbb{F}, \partial=0)$, ie an algebra map satisfying $\varepsilon(1)=1$ and $\varepsilon \circ \partial=0$. If $\varepsilon(c)=0$ for $|c| \neq 0, c \in \mathcal{Q}(\Lambda)$, we say that $\varepsilon$ is graded. If $(\mathcal{A}(\Lambda), \partial)$ admits an augmentation (which is not always the case), then we can follow the linearization procedure due to Chekanov to produce a complex spanned as a vector space by the Reeb chords. We first define a tame automorphism $\sigma_{\varepsilon}:(\mathcal{A}, \partial) \rightarrow(\mathcal{A}, \partial)$ with $\sigma_{\varepsilon}(c)=c+\varepsilon(c)$. Then we define $\left(\mathcal{C}(\Lambda), \partial^{\varepsilon}:=\left(\sigma_{\varepsilon} \circ \partial \circ \sigma_{\varepsilon}^{-1}\right)_{1}\right)$, where $\mathcal{C}(\Lambda)$ is the $\mathbb{F}$-vector space spanned by the elements of $\mathcal{Q}(\Lambda)$. The homology of $\left(\mathcal{C}(\Lambda), \partial^{\varepsilon}\right)$ is called the linearized Legendrian contact homology of $\Lambda$.

Observe that an augmentation can be seen as a 1-dimensional linear representation of $\mathcal{A}(\Lambda)$ which satisfies the additional property that $\varepsilon \circ \partial=0$. A $k$-dimensional linear representation $\rho: \mathcal{A}(\Lambda) \rightarrow \mathrm{M}_{k}(\mathbb{F})$ is called graded if $\rho(c)=0$ for $|c| \neq 0, c \in \mathcal{Q}(\Lambda)$. We will be interested in such representations satisfying the additional condition $\rho \circ \partial=0$. 
Assume that we are given a Legendrian submanifold $\Lambda \subset(P \times \mathbb{R}, d z+\theta)$ with Chekanov-Eliashberg DGA $(\mathcal{A}(\Lambda), \partial)$. $\mathrm{Ng}$ [24] defined the so-called characteristic algebra of $\Lambda$, which is given by $\mathcal{C}_{\Lambda}:=\mathcal{A}(\Lambda) / I$, where $I$ denotes the two-sided ideal of $\mathcal{A}(\Lambda)$ generated by $\{\partial(c)\}_{c \in \mathcal{Q}(\Lambda)}$. If two submanifolds $\Lambda$ and $\Lambda^{\prime}$ are Legendrian isotopic, then $\mathcal{C}_{\Lambda}$ and $\mathcal{C}_{\Lambda^{\prime}}$ become isomorphic after stabilizations by free products with suitable finitely generated free algebras, as follows from [24, Theorem 3.4] together with [10, Theorem 1.1].

Note that there is a one-to-one correspondence between (graded) linear representations $\rho: \mathcal{A}(\Lambda) \rightarrow \mathbf{M}_{k}(\mathbb{F})$ satisfying $\rho \circ \partial=0$ and (graded) linear representations $\mathcal{C}_{\Lambda} \rightarrow \mathrm{M}_{k}(\mathbb{F})$. Again, [24, Theorem 3.4] together with [10, Theorem 1.1] shows the following important invariance result:

Proposition 1.1 The property of having a characteristic algebra admitting a (graded) $k-$ dimensional representation is invariant under Legendrian isotopy and independent of the choice of almost complex structure used in the definition of the Chekanov-Eliashberg algebra.

The front $S^{1}$-spinning is a procedure defined by Ekholm, Etnyre and Sullivan [8] which, given a Legendrian submanifold $\Lambda \subset \mathbb{C}^{n} \times \mathbb{R}$, produces a Legendrian submanifold $\Sigma_{S^{1}} \Lambda \subset \mathbb{C}^{n+1} \times \mathbb{R}$ diffeomorphic to $\Lambda \times S^{1}$. The second author [17] generalized this construction to a notion of the front $S^{m}$-spinning for $m \in \mathbb{N}$. Given a Legendrian submanifold $\Lambda \subset \mathbb{C}^{n} \times \mathbb{R}$, this construction produces a Legendrian submanifold $\Sigma_{S^{m}} \Lambda \subset \mathbb{C}^{n+m} \times \mathbb{R}$ diffeomorphic to $\Lambda \times S^{m}$. Observe that the front $S^{m}$-spinning construction can be seen as a particular case of the Legendrian product construction defined by Lambert-Cole [21].

\subsection{Algebraic background}

In this section, we recall several definitions from non-commutative ring theory that will become useful later.

Definition 1.2 A ring $R$ satisfies

- the invariant basis number $(I B N)$ property if the left-modules $R^{n}$ and $R^{m}$ are isomorphic if and only if $m=n$;

- the rank property if from the existence of an epimorphism $R^{n} \rightarrow R^{m}$ of free left modules it follows that $n \geq m$;

- the strong rank property if from the existence of monomorphism $R^{n} \rightarrow R^{m}$ of free left modules it follows that $n \leq m$.

The following three remarks, which describe basic properties of these three conditions, can all be derived using elementary algebra. 
Remark 1.3 Let $f: R \rightarrow S$ be a (unital) ring homomorphism. If $S$ satisfies the rank property (the IBN property), then $R$ satisfies the rank property (the IBN property). Unfortunately, the same principle does not hold for the strong rank property in general.

Remark 1.4 For a unital ring we have the implications

$$
\text { strong rank property } \Longrightarrow \text { rank property } \Longrightarrow \text { IBN property. }
$$

In general, none of the reversed implications are true.

Remark 1.5 A ring $R$ fails to satisfy the rank property if and only if there exist natural numbers $m>n$ and matrices $A, B$ over $R$ of sizes $m \times n$ and $n \times m$, respectively, such that $A B=I_{m}$.

\subsection{Results}

Many important techniques in symplectic and contact topology are designed to capture quantitative properties of dynamical systems induced by, say, a Hamiltonian flow or a Reeb flow. The problem of finding closed orbits of a Hamiltonian vector field and a Reeb vector field can in turn be related to the problems of finding intersections between Lagrangian submanifolds and Reeb chords on Legendrian submanifolds, respectively. Several examples of rigidity phenomena have been established in different settings, where the numbers of such intersection points, or chords, are strictly greater than the numbers predicted by topology alone. Often these estimates are proven using different variants of Floer homology.

1.3.1 The first lower bounds Gromov's theorem [18, Theorem 2.3. $\left.B_{1}\right]$ implies that any horizontally displaceable Legendrian submanifold must have at least one Reeb chord. Note that this estimate is already highly non-trivial in general.

In even dimensions, there is a lower bound obtained from topology alone. Assume that we are given a chord-generic, horizontally displaceable, orientable Legendrian submanifold $\Lambda \subset P \times \mathbb{R}$, where $\operatorname{dim} P=2 n$ and $n=2 k$ is even. The Whitney self-intersection index of $\Pi_{L}(\Lambda)$ is equal to $(-1)^{k+1} \frac{1}{2} \chi(\Lambda)$, as follows from purely topological considerations; see Audin [2, Proposition 0.4] for $P=\mathbb{C}^{n}$. In particular, $\Pi_{L}(\Lambda)$ has at least $\frac{1}{2}|\chi(\Lambda)|$ double points, and hence $|\mathcal{Q}(\Lambda)| \geq \frac{1}{2}|\chi(\Lambda)|$. In the case when $P=\mathbb{C}^{2 k}$, [8, Proposition 3.2(2)] shows moreover that $\operatorname{tb}(\Lambda)=(-1)^{k+1} \frac{1}{2} \chi(\Lambda)$. 
1.3.2 Arnold's inequality For some time it was expected that a horizontally displaceable, chord-generic, Legendrian submanifold would have at least as many Reeb chords as half the sum of its Betti numbers. This type of inequality is in line with other conjectures due to Arnold [1], and it is usually referred to as Arnold's inequality or an Arnold-type inequality. This inequality is however not fulfilled in general; we refer to Section 1.3.3 for references to counter-examples belonging to the so-called flexible side of contact geometry.

On the other hand, it has been shown that the inequality is fulfilled for certain natural Legendrian isotopy classes. In the case when the Chekanov-Eliashberg algebra of $\Lambda \subset P \times \mathbb{R}$ admits an augmentation, the inequality was originally proven by Ekholm, Etnyre and Sullivan [9]. A "graded" refinement of this result is also a consequence of the (considerably stronger) duality result for linearized Legendrian contact homology due to Ekholm, Etnyre and Sabloff [7]. In this paper, we show that the requirement of having an augmentation can be relaxed considerably in the proof of the above inequality.

Theorem 1.6 Let $\Lambda \subset P \times \mathbb{R}$ be an $n$-dimensional horizontally displaceable Legendrian submanifold which is chord-generic. If its characteristic algebra $\mathcal{C}_{\Lambda}$ admits a $k$-dimensional representation $\rho: \mathcal{C}_{\Lambda} \rightarrow \mathrm{M}_{k}(\mathbb{F})$ for some field $\mathbb{F}$, then the following inequality holds:

$$
\frac{1}{2} \sum_{i \in I} b_{i} \leq \sum_{i \in I} c_{i},
$$

where $b_{j}:=\operatorname{dim}_{\mathbb{F}} H_{j}(\Lambda ; \mathbb{F}), c_{j}$ is the number of Reeb chords on $\Lambda$ of grading $j$ and $I \in\{2 \mathbb{Z}, \mathbb{Z} \backslash 2 \mathbb{Z}\}$.

In addition, if $\rho$ is a graded representation, then the following refinement of the previous inequality holds:

$$
b_{i} \leq c_{i}+c_{n-i}
$$

for all $i$ satisfying $0 \leq i \leq n$.

Remark 1.7 In the case when char $\mathbb{F} \neq 2$, we must make the additional assumption that $\Lambda$ is spin in order for its Chekanov-Eliashberg algebra to be well-defined with coefficients in $\mathbb{F}$.

To provide interesting examples of Legendrian submanifolds satisfying the assumptions of the theorem we will proceed as follows. In the literature, there are many computations of Chekanov-Eliashberg algebras of Legendrian knots in $\mathbb{C} \times \mathbb{R}$. We provide higher-dimensional examples by using the front $S^{m}$-spinning construction. 
The Chekanov-Eliashberg algebra of the $S^{1}$-spinning of a 1-dimensional Legendrian knot was computed in terms of the Chekanov-Eliashberg algebra of the original knot by Ekholm and Kálmán [12]. In Section 3, we provide a part of this computation in the general case. Even though we do not compute the full Chekanov-Eliashberg algebra of the $S^{m}$-spinning of a general Legendrian submanifold $\Lambda$, we are able to establish the following result:

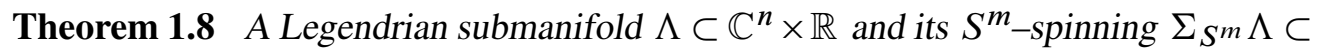
$\mathbb{C}^{n+m} \times \mathbb{R}$ satisfy the following relations:

(1) The Chekanov-Eliashberg algebra of $\Sigma_{S^{m}} \Lambda$ admits a (graded) augmentation in a unital ring $R$ if and only if that of $\Lambda$ admits a (graded) augmentation in $R$.

(2) The characteristic algebra of $\Sigma_{S^{m}} \Lambda$ admits a (graded) $k$-dimensional representation if and only if that of $\Lambda$ admits a (graded) $k$-dimensional representation.

(3) The Chekanov-Eliashberg algebra of $\Sigma_{S^{m}} \Lambda$ is acyclic if and only if that of $\Lambda$ is acyclic.

Sivek [29] has provided examples of Legendrian knots in $\mathbb{C} \times \mathbb{R}$ whose characteristic algebras admit 2-dimensional, but not 1-dimensional, representations over $\mathbb{Z}_{2}$. In Section 4, we apply the front $S^{m}$-spinning construction to these knots and other knots constructed from them and, using Theorem 1.8, we prove the following:

Theorem 1.9 Fix a product $S:=S^{m_{1}} \times \cdots \times S^{m_{s}}$ of spheres. There exists an infinite family of Legendrian embeddings $\Lambda_{i} \subset \mathbb{C}^{1+m_{1}+\cdots+m_{s}} \times \mathbb{R}, i=1,2, \ldots$, of $S^{1} \times S$ which are in pairwise different Legendrian isotopy classes and whose characteristic algebras all admit finite-dimensional matrix representations over $\mathbb{Z}_{2}$, but whose Chekanov-Eliashberg algebras do not admit augmentations to unital commutative rings. In particular, Theorem 1.6 can be applied to these Legendrian submanifolds.

Observe that the condition that there exists a finite-dimensional representation of $\mathcal{C}_{\Lambda}$ over a field $\mathbb{F}$ is a natural condition that can be used to define the notion of rank for free $\mathcal{C}_{\Lambda}-\mathcal{C}_{\Lambda^{\prime}}$-bimodules in the case when $\Lambda^{\prime}$ is Legendrian isotopic to $\Lambda$. It also allows us to write rank inequalities for long exact sequences of such free finite-rank $\mathcal{C}_{\Lambda}-\mathcal{C}_{\Lambda^{\prime}}-$ bimodules. These rank inequalities play a crucial role in the proof of Theorem 1.6. We do not know if this restriction on $\mathcal{C}_{\Lambda}$ can be weakened. On the other hand, in Section 5 we show that there are examples of Legendrian submanifolds of $\mathbb{C}^{n} \times \mathbb{R}$ with a "wild" behavior of $\mathcal{C}_{\Lambda}$. More precisely, there are $n$-dimensional Legendrian submanifolds $\Lambda$ with non-trivial $\mathcal{C}_{\Lambda}$ for which there exists a monomorphism (epimorphism) from a free $\mathcal{C}_{\Lambda}-\mathcal{C}_{\Lambda^{\prime}}$-bimodule of rank $k$ to a free $\mathcal{C}_{\Lambda}-\mathcal{C}_{\Lambda^{\prime}}$-bimodule of rank $l$, where $k>l(k<l)$. In other words, we prove the following: 
Theorem 1.10 Given any $n \geq 1$, there exists a Legendrian submanifold $\Lambda \subset \mathbb{C}^{n} \times \mathbb{R}$ whose characteristic algebra $\mathcal{C}_{\Lambda} \neq 0$ does not satisfy the rank property.

This result shows that it is unlikely that the method in the proof of Theorem $1.6 \mathrm{can}$ be used to prove an Arnold-type inequality for a general horizontally displaceable Legendrian submanifold having a Chekanov-Eliashberg algebra which is not acyclic.

1.3.3 A failure of Arnold's inequality There are examples of horizontally displaceable Legendrian submanifolds $\Lambda \subset P \times \mathbb{R}$ for which Arnold's inequality is not satisfied. Observe that all known examples have an acyclic Chekanov-Eliashberg algebra.

The first example of a Legendrian submanifold for which Arnold's inequality does not hold was provided by Sauvaget [28], who constructed a genus-two Legendrian surface in $\mathbb{C}^{2} \times \mathbb{R}$ having only one transverse Reeb chord.

Loose Legendrian submanifolds is a class of Legendrian submanifolds defined by Murphy [23], who showed they satisfy an h-principle. Since these submanifolds belong to the flexible domain of contact geometry, one does not expect them to satisfy any rigidity phenomena. Observe that the Chekanov-Eliasbherg algebra of a loose Legendrian submanifold is acyclic (with or without Novikov coefficients). Using this h-principle, together with the h-principle for exact Lagrangian caps as shown in [14], Ekholm, Eliashberg, Murphy and Smith [6] provided many examples of exact Lagrangian immersions with few double points. We present here a weaker form of their result.

Theorem 1.11 [6] Suppose that $\Lambda$ is a smooth closed $n$-dimensional manifold for which $T \Lambda \otimes \mathbb{C}$ is a trivial complex bundle. There exists a loose, horizontally displaceable, chord-generic, Legendrian embedding $\Lambda \subset \mathbb{C}^{n} \times \mathbb{R}$ satisfying

$$
\begin{cases}1 \leq|\mathcal{Q}(\Lambda)| \leq 2 & \text { if } n \text { is odd, } \\ |\mathcal{Q}(\Lambda)|=\frac{1}{2}|\chi(\Lambda)| & \text { if } n \text { is even and } \chi(\Lambda)<0, \\ \frac{1}{2}|\chi(\Lambda)| \leq|\mathcal{Q}(\Lambda)| \leq \frac{1}{2}|\chi(\Lambda)|+2 & \text { if } n \text { is even and } \chi(\Lambda)>0 .\end{cases}
$$

Some rather basic algebraic considerations show the following (very) slight improvement of the lower bound in the case when a Legendrian submanifold has a non-acyclic Chekanov-Eliashberg algebra.

Proposition 1.12 Suppose that $\Lambda \subset P \times \mathbb{R}$ is a horizontally displaceable, chord-generic $n$-dimensional Legendrian submanifold whose characteristic algebra is non-trivial, but does not admit any finite-dimensional representations. It follows that

$$
|\mathcal{Q}(\Lambda)| \geq 3
$$


Moreover, if $n=2 k$, we have the bound

$$
|\mathcal{Q}(\Lambda)| \geq \frac{1}{2}|\chi(\Lambda)|+2
$$

under the additional assumptions that $\Lambda$ is orientable and either

(1) $\chi(\Lambda) \geq 0$, or

(2) $\mu(\Lambda)=0$ and all generators have non-negative grading.

\section{Acknowledgements}

This work was partially supported by the ERC Starting Grant of Frédéric Bourgeois StG-239781-ContactMath and by the ERC Advanced Grant LDTBud. The authors are deeply grateful to Yakov Eliashberg, John Etnyre and Michael Sullivan for helpful conversations and interest in their work. In addition, Golovko would like to thank the Simons Center, where part of this article was written, for its hospitality. Finally, the authors are grateful to the referee for many valuable comments and suggestions.

\section{Proof of Theorem 1.6}

The proof uses the same construction and idea as Ekholm, Etnyre and Sabloff [7]. In other words, we consider the two-copy link $\Lambda \cup \Lambda^{\prime}$ constructed as follows. First, identify a neighborhood of $\Lambda$ with a neighborhood of the zero-section $\Lambda \subset J^{1}(\Lambda)$ using the standard neighborhood theorem for Legendrian submanifolds. Using this identification, the section $j^{1} f \subset J^{1}(\Lambda)$ can be considered as a Legendrian submanifold $\Lambda^{\prime \prime} \subset P \times \mathbb{R}$, where $f: \Lambda \rightarrow \mathbb{R}$ is a $C^{2}$-small Morse function. $\Lambda^{\prime}$ is now obtained from $\Lambda^{\prime \prime}$ by a translation sufficiently far in the positive Reeb direction, so that there are no Reeb chords starting on $\Lambda^{\prime}$ and ending on $\Lambda$. Observe that $\Pi_{L}\left(\Lambda^{\prime}\right)$ may still be assumed to be arbitrarily close to $\Pi_{L}(\Lambda)$ in $C^{1}$-norm, and hence that there is a canonical bijective correspondence $\mathcal{Q}(\Lambda) \simeq \mathcal{Q}\left(\Lambda^{\prime}\right)$ of Reeb chords.

By topological considerations of the disks involved in the Chekanov-Eliashberg algebra of $\Lambda \cup \Lambda^{\prime}$, we see that there is a filtration

$$
\left(\mathcal{A}\left(\Lambda \cup \Lambda^{\prime}\right), \partial\right) \supset \cdots \supset\left(\mathcal{A}\left(\Lambda \cup \Lambda^{\prime}\right)^{1}, \partial\right) \supset\left(\mathcal{A}\left(\Lambda \cup \Lambda^{\prime}\right)^{0}, \partial\right),
$$

where $\left(\mathcal{A}\left(\Lambda \cup \Lambda^{\prime}\right)^{i}, \partial\right)$ is spanned by words containing at most $i$ letters corresponding to mixed chords having starting point on $\Lambda$ and endpoint on $\Lambda^{\prime}$. We can thus define the quotient complex

$$
\left(\mathcal{A}\left(\Lambda, \Lambda^{\prime}\right), \partial\right):=\left(\mathcal{A}\left(\Lambda \cup \Lambda^{\prime}\right)^{1}, \partial\right) / \mathcal{A}\left(\Lambda \cup \Lambda^{\prime}\right)^{0},
$$


which we identify with the vector space over $\mathbb{F}$ spanned by words of the form $\boldsymbol{a} \boldsymbol{c} \boldsymbol{b}$, where $\boldsymbol{a}$ is a word of Reeb chords on $\Lambda, c$ is a Reeb chord starting on $\Lambda$ and ending on $\Lambda^{\prime}$, and $\boldsymbol{b}$ is a word of Reeb chords on $\Lambda^{\prime}$. In fact, this $\mathbb{F}$-vector space is naturally a free $\mathcal{A}(\Lambda)-\mathcal{A}\left(\Lambda^{\prime}\right)$-bimodule spanned by the set $\mathcal{Q}\left(\Lambda, \Lambda^{\prime}\right)$ of Reeb chords starting on $\Lambda$ and ending on $\Lambda^{\prime}$. However, the differential is in general not a morphism of bimodules.

From the existence of $\rho: \mathcal{C}_{\Lambda} \rightarrow \mathbf{M}_{k}(\mathbb{F})$ and the fact that $\Lambda$ and $\Lambda^{\prime}$ are Legendrian isotopic, it follows from Proposition 1.1 that there exists a linear representation $\rho^{\prime}: \mathcal{C}_{\Lambda^{\prime}} \rightarrow \mathrm{M}_{k}(\mathbb{F})$ as well. Let $\mathcal{C}\left(\Lambda, \Lambda^{\prime}\right)$ denote the free $\mathcal{C}_{\Lambda}-\mathcal{C}_{\Lambda^{\prime}}$-bimodule generated by $\mathcal{Q}\left(\Lambda, \Lambda^{\prime}\right)$. Similarly, let $\mathcal{C}_{\rho}\left(\Lambda, \Lambda^{\prime}\right)$ denote the free $\mathrm{M}_{k}(\mathbb{F})-\mathrm{M}_{k}(\mathbb{F})$-bimodule generated by $\mathcal{Q}\left(\Lambda, \Lambda^{\prime}\right)$. Observe that the compositions

$$
\begin{gathered}
\mathcal{A}(\Lambda) \stackrel{\pi_{\Lambda}}{\longrightarrow} \mathcal{C}_{\Lambda} \stackrel{\rho}{\longrightarrow} \mathrm{M}_{k}(\mathbb{F}), \\
\mathcal{A}\left(\Lambda^{\prime}\right) \stackrel{\pi_{\Lambda^{\prime}}}{\longrightarrow} \mathcal{C}_{\Lambda^{\prime}} \stackrel{\rho^{\prime}}{\longrightarrow} \mathrm{M}_{k}(\mathbb{F}),
\end{gathered}
$$

where $\pi_{\Lambda}$ and $\pi_{\Lambda^{\prime}}$ are the quotient projections, induce a commutative diagram

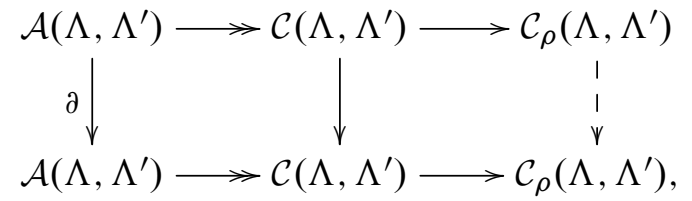

where all maps are $\mathbb{F}$-linear and the horizontal maps are the induced natural $\mathcal{A}(\Lambda)$ $\mathcal{A}\left(\Lambda^{\prime}\right)$-bimodule morphisms. The middle vertical map is a uniquely determined morphism of $\mathcal{C}_{\Lambda^{-}}-\mathcal{C}_{\Lambda^{\prime}}$-bimodules induced by the differential $\partial$, as follows from the Leibniz rule

$$
\partial(\boldsymbol{a} c \boldsymbol{b})=\partial_{\Lambda}(\boldsymbol{a}) c \boldsymbol{b}+(-1)^{|\boldsymbol{a} c|} \boldsymbol{a} c \partial_{\Lambda^{\prime}}(\boldsymbol{b})+(-1)^{|\boldsymbol{a}|} \boldsymbol{a} \partial(c) \boldsymbol{b}
$$

together with the fact that $\partial_{\Lambda}(\boldsymbol{a})$ and $\partial_{\Lambda^{\prime}}(\boldsymbol{b})$ are in the kernels of the projection to the respective characteristic algebras. It follows that the rightmost vertical map is uniquely determined by the requirement of being an $\mathrm{M}_{k}(\mathbb{F})-\mathrm{M}_{k}(\mathbb{F})$-bimodule morphism making the diagram commutative.

Remark 2.1 In the case when $k=1$, the rightmost vertical map is simply the linearized differential with respect to the augmentation $\epsilon: \mathcal{A}\left(\Lambda \cup \Lambda^{\prime}\right) \rightarrow \mathbb{F}$ that is defined as follows: $\epsilon$ vanishes on generators corresponding to chords starting on $\Lambda$ and ending on $\Lambda^{\prime}$, while it takes the value $\rho \circ \pi_{\Lambda}$ and $\rho^{\prime} \circ \pi_{\Lambda^{\prime}}$ on generators corresponding to chords on $\Lambda$ and $\Lambda^{\prime}$, respectively. 
The assumption of horizontal displaceability implies that there is a Legendrian isotopy $\Lambda_{t} \cup \Lambda_{t}^{\prime}$ for which $\Lambda_{0} \cup \Lambda_{0}^{\prime}=\Lambda \cup \Lambda^{\prime}$ and such that there are no chords between $\Lambda_{1}$ and $\Lambda_{1}^{\prime}$. The invariance proof of Ekholm, Etnyre and Sullivan [10] implies that, after a finite number of stabilizations by the direct sum of a trivial complex with two generators, the complex $\left(\mathcal{A}\left(\Lambda, \Lambda^{\prime}\right), \partial\right)$ is isomorphic to the stabilization of a trivial complex. It follows that the same is true for the complex $\left(\mathcal{C}_{\rho}\left(\Lambda, \Lambda^{\prime}\right), \partial\right)$. In particular, the latter complex is acyclic as well.

Observe that there is a natural isomorphism

$$
\Phi: \mathrm{M}_{k}(\mathbb{F}) \otimes \mathrm{M}_{k}(\mathbb{F})^{\mathrm{op}} \rightarrow \mathrm{M}_{k^{2}}(\mathbb{F})
$$

of $\mathbb{F}$-algebras determined by taking the values $\Phi(A \otimes B)(v \otimes w):=A(v) \otimes B^{T}(w)$ and then extended by linearity for $v, w \in \mathbb{F}^{k}$ and $A, B \in \mathrm{M}_{k}(\mathbb{F})$, where we have used an identification $\mathbb{F}^{k} \otimes \mathbb{F}^{k} \simeq \mathbb{F}^{k^{2}}$. The considerations of the set $\mathcal{Q}\left(\Lambda, \Lambda^{\prime}\right)$ in [7, Section 3.1] show that

$$
\mathcal{C}_{\rho}\left(\Lambda, \Lambda^{\prime}\right)=\mathcal{Q} \oplus \mathcal{C} \oplus \mathcal{P}
$$

where

$$
\mathcal{Q}=\mathrm{M}_{k^{2}}(\mathbb{F})^{\oplus \mathcal{Q}(\Lambda)}, \quad \mathcal{C}=\mathrm{M}_{k^{2}}(\mathbb{F})^{\oplus \operatorname{Crit}(f)}, \quad \mathcal{P}=\mathrm{M}_{k^{2}}(\mathbb{F})^{\oplus \mathcal{Q}(\Lambda)} .
$$

Here, $\mathcal{Q}$ and $\mathcal{P}$ are both generated by subsets of $\mathcal{Q}\left(\Lambda, \Lambda^{\prime}\right)$ which are in canonical bijection with $\mathcal{Q}(\Lambda)$, and $\mathcal{C}$ is generated by Reeb chords which are in canonical bijection with the critical points of $f$.

Moreover, using appropriate choices of Maslov potentials, a generator $q_{c} \in \mathcal{Q}$ corresponding to the chord $c \in \mathcal{Q}(\Lambda)$ is graded by $\left|q_{c}\right|=|c|$, a generator $c_{x} \in \mathcal{C}$ corresponding to the critical point $x$ is graded by $\left|c_{x}\right|=\operatorname{index}_{\operatorname{Morse}}(x)-1$, while a generator $q_{c} \in \mathcal{P}$ corresponding to the chord $c \in \mathcal{Q}(\Lambda)$ is graded by $\left|q_{c}\right|=-|c|+n-2$. Finally, we may assume that the actions of the generators in $\mathcal{Q}$ are strictly greater than the actions of the generators in $\mathcal{C}$, which, in turn, are strictly greater than the actions of the generators in $\mathcal{P}$.

The analysis in [7, Theorem 3.6] moreover shows that, for a suitable choice of almost complex structure and metric on $\Lambda$, the differential with respect to the above decomposition is of the form

$$
\partial=\left(\begin{array}{ccc}
* & 0 & 0 \\
* & \partial_{f} & 0 \\
* & * & *
\end{array}\right),
$$

where $\partial_{f}$ is the Morse differential induced by a Morse-Smale pair $(f, g)$ (where the coefficients have been taken in $\mathrm{M}_{k^{2}}(\mathbb{F})$ ). 
The inclusion of subcomplexes

$$
\mathcal{P} \subset \mathcal{C} \oplus \mathcal{P} \subset\left(\mathcal{C}_{\rho}\left(\Lambda, \Lambda^{\prime}\right), \partial\right)
$$

induces the long exact sequence

$$
\cdots \rightarrow H_{\bullet}(\mathcal{P}) \rightarrow H_{\bullet}(\mathcal{C} \oplus \mathcal{P}) \rightarrow H_{\bullet+1}^{\text {Morse }}\left(f ; \mathrm{M}_{k^{2}}(\mathbb{F})\right) \rightarrow H_{\bullet-1}(\mathcal{P}) \rightarrow \cdots
$$

in homology. Since the acyclicity of $\left(\mathcal{C}_{\rho}\left(\Lambda, \Lambda^{\prime}\right), \partial\right)$ implies that

$$
H_{\bullet}(\mathcal{C} \oplus \mathcal{P}) \simeq H_{\bullet+1}(\mathcal{Q})
$$

we obtain the long exact sequence

$$
\cdots \rightarrow H_{\bullet}(\mathcal{P}) \rightarrow H_{\bullet+1}(\mathcal{Q}) \rightarrow H_{\bullet+1}^{\mathrm{Morse}}\left(f ; \mathrm{M}_{k^{2}}(\mathbb{F})\right) \rightarrow H_{\bullet-1}(\mathcal{P}) \rightarrow \cdots .
$$

Exactness and the fact that all modules in the long exact sequence are finite-dimensional $\mathbb{F}$-vector spaces imply that we have the inequality

$$
\operatorname{dim}_{\mathbb{F}} H_{i}^{\operatorname{Morse}}\left(f ; \mathbf{M}_{k^{2}}(\mathbb{F})\right) \leq \operatorname{dim}_{\mathbb{F}} H_{i-2}(\mathcal{P})+\operatorname{dim}_{\mathbb{F}} H_{i}(\mathcal{Q}),
$$

where we can compute the left-hand side to be

$$
\operatorname{dim}_{\mathbb{F}} H_{i}^{\text {Morse }}\left(f ; \mathrm{M}_{k^{2}}(\mathbb{F})\right)=k^{4} \operatorname{dim}_{\mathbb{F}} H_{i}^{\mathrm{Morse}}(f ; \mathbb{F})=k^{4} b_{i},
$$

since we are using field coefficients and $\operatorname{dim}_{\mathbb{F}} \mathrm{M}_{k^{2}}(\mathbb{F})=k^{4}$. On the other hand, we also have the bounds

$$
\begin{aligned}
\operatorname{dim}_{\mathbb{F}} H_{i}(\mathcal{Q}) & \leq \operatorname{dim}_{\mathbb{F}} \mathcal{Q}_{i}=k^{4} c_{i}, \\
\operatorname{dim}_{\mathbb{F}} H_{i-2}(\mathcal{P}) & \leq \operatorname{dim}_{\mathbb{F}} \mathcal{P}_{i-2}=k^{4} c_{n-i},
\end{aligned}
$$

from which the sought inequality follows.

\section{A partial computation of the Chekanov-Eliashberg algebra for an $S^{m}$-spun Legendrian}

In the following we let $\Lambda \subset P \times \mathbb{R}$ be an arbitrary closed chord-generic Legendrian submanifold of the contactization of a Liouville domain. We prove here the following relationship between the Chekanov-Eliashberg algebra of the Legendrian submanifold $S^{m} \times \Lambda \subset T^{*} S^{m} \times P \times \mathbb{R}$ and the Chekanov-Eliashberg algebra of $\Lambda$ :

Theorem 3.1 For a suitable almost complex structure on $T^{*} S^{m} \times P$ and a suitable Legendrian chord-generic perturbation $L$ of $S^{m} \times \Lambda \subset T^{*} S^{m} \times P \times \mathbb{R}$, there is a canonical inclusion

$$
\iota:\left(\mathcal{A}_{\bullet}(\Lambda), \partial_{\Lambda}\right) \rightarrow\left(\mathcal{A}_{\bullet}(L), \partial_{L}\right)
$$


of unital DGAs which, moreover, can be left-inverted by a unital DGA morphism

$$
\pi:\left(\mathcal{A}_{\bullet}(L), \partial_{L}\right) \rightarrow\left(\mathcal{A}_{\bullet}(\Lambda), \partial_{\Lambda}\right)
$$

If $P=\mathbb{C}^{n}$, then the same is true for the corresponding Legendrian submanifold obtained by an inclusion induced by an exact symplectic embedding

$$
\left(T^{*} S^{m} \times T \mathbb{R}^{n}, d \theta_{S^{m}} \oplus d \theta_{\mathbb{R}^{n}}\right) \hookrightarrow\left(T \mathbb{R}^{n+m}, d \theta_{\mathbb{R}^{n+m}}\right),
$$

ie the $S^{m}$-spinning $\Sigma_{S^{m}} \Lambda \subset J^{1} \mathbb{R}^{n+m}$ of $\Lambda$.

Remark 3.2 Ekholm and Kálmán [12] computed the full Chekanov-Eliashberg algebra of $\Sigma_{S^{1}} \Lambda$ in terms of the Chekanov-Eliashberg algebra of $\Lambda$ under the additional assumption that $\operatorname{dim} \Lambda=1$.

Note that Theorem 1.8 immediately follows from Theorem 3.1 using elementary algebraic considerations.

We postpone the proof of Theorem 3.1 to Section 3.5. The idea is to find a geometric correspondence between the pseudo-holomorphic disks defining the differential $\partial_{\Lambda}$ and those defining $\partial_{L}$. This correspondence will be induced by the canonical projection map $T^{*} S^{m} \times P \rightarrow P$ for some sufficiently symmetric perturbation of $L$ and choice of almost complex structure. Even though not all disks in the definition of $\partial_{L}$ are transversely cut out for these choices, we will show that the parts of the differential needed to deduce the above result still will be determined by transversely cut-out disks.

\subsection{Constructing the perturbation $L$ of $S^{m} \times \Lambda$}

Consider the two antipodal points

$$
N=(0, \ldots, 0,1) \text { and } S=(0, \ldots, 0,-1)
$$

on the unit sphere $S^{m} \subset \mathbb{R}^{m+1}$. We will take $g: S^{m} \rightarrow[0,1]$ to be the Morse function with two critical points obtained as the restriction of $\frac{1}{2}\left(1+x_{m+1}\right)$, where $x_{m+1}$ denotes the last standard coordinate on $\mathbb{R}^{m+1}$. The critical points of $g$ are obviously $S$ and $N$ with critical values $g(S)=0$ and $g(N)=1$, respectively. Moreover, we have $g \circ r=g$ for any $r \in O(m) \subset O(m+1)$, where we use $O(m)$ to denote the orthogonal transformations of $\mathbb{R}^{m+1}$ that fix $\{N, S\}$ pointwise.

In the following we will let $h: \Lambda \rightarrow \mathbb{R}$ denote the $z$-coordinate restricted to $\Lambda \subset P \times \mathbb{R}$. In other words, $-h$ is a primitive of $\theta$ pulled back to $\Lambda$. Consider the so-called 
Liouville flow $\phi^{t}: P \rightarrow P$ with respect to $\theta$, which is determined by the property that $\left(\phi^{t}\right)^{*}(\theta)=e^{t} \theta$. One constructs a Legendrian isotopy $L_{t} \subset T^{*} S^{m} \times P$ parametrized by

$$
\begin{aligned}
S^{m} \times \Lambda & \rightarrow T^{*} S^{m} \times P \times \mathbb{R}, \\
(\boldsymbol{q}, x) & \mapsto\left(-h(x) d e^{\operatorname{tg}(\boldsymbol{q})}, \phi^{\operatorname{tg}(\boldsymbol{q})}(x), h(x) e^{\operatorname{tg}(\boldsymbol{q})}\right),
\end{aligned}
$$

for which $L_{0}=S^{m} \times \Lambda$. We will be interested in the Legendrian embedding $L:=L_{\epsilon}$ for some sufficiently small $\epsilon>0$, which thus may be assumed to be arbitrarily $C^{1}$-close to the Legendrian embedding $S^{m} \times \Lambda$.

Let

$$
\pi_{T^{*} S^{m}}: T^{*} S^{m} \times P \rightarrow T^{*} S^{m}
$$

denote the canonical projection. Under the assumption that $\Lambda$ is chord-generic, it follows that $L$ is chord-generic as well. The Reeb chords on $L$ are all contained in either $\pi_{T^{*} S^{m}}^{-1}(S)$ or $\pi_{T^{*} S^{m}}^{-1}(N)$, and we use $\mathcal{Q}_{S}$ and $\mathcal{Q}_{N}$ to denote the respective sets of Reeb chords. Observe that there is a canonical bijection between each of these subsets of Reeb chords on $L$ with the Reeb chords on $\Lambda$. More precisely, one can say the following:

Lemma 3.3 The canonical bijection $\mathcal{Q}_{S} \simeq \mathcal{Q}(\Lambda)$ preserves both the action and index of the chords while, for the canonical bijection $\mathcal{Q}_{N} \simeq \mathcal{Q}(\Lambda)$, the action is multiplied by $e^{\epsilon}$ and the grading is increased by $m$.

\subsection{A suitable almost complex structure}

Here we construct an almost complex structure $J$ on $T^{*} S^{m} \times P$ that suits our needs.

3.2.1 An integrable almost complex structure on $T^{*} S^{m}$ Let $i$ denote the standard complex structure on

$$
A_{1}^{m}=\left\{z_{1}^{2}+\cdots+z_{m+1}^{2}=1\right\} \subset \mathbb{C}^{m+1} .
$$

We will use the following explicit identification between $T^{*} S^{m}$ and $A_{1}^{m}$. Using the round metric together with the canonical embedding $S^{m} \subset \mathbb{R}^{m+1}$, we identify $(\boldsymbol{q}, \boldsymbol{p}) \in T^{*} S^{m}$ with the tangent vector $v_{\boldsymbol{p}} \in T_{\boldsymbol{q}} S^{m} \subset T_{\boldsymbol{q}} \mathbb{R}^{m+1}$. We now consider the identification

$$
\begin{aligned}
\Psi: T^{*} S^{m} & \rightarrow A_{1}^{m} \subset \mathbb{C}^{m+1}, \\
(\boldsymbol{q}, \boldsymbol{p}) & \mapsto \sqrt{1+\left\|v_{\boldsymbol{p}}\right\|^{2}} \boldsymbol{q}-i v_{\boldsymbol{p}} .
\end{aligned}
$$

It is readily checked that $\eta(x+i \boldsymbol{y}):=\sqrt{1+\|\boldsymbol{y}\|^{2}}$ is strictly plurisubharmonic on $\left(\mathbb{C}^{m+1}, i\right)$, ie that $-d(d \eta \circ i)$ is a Kähler form, and that moreover

$$
\Psi^{*}(-d \eta \circ i)=\theta_{S^{m}},
$$


where $\theta_{S^{m}}$ is the Liouville form on $T^{*} S^{m}$.

Observe that the linear action of $r \in O(m+1)$ on $\mathbb{R}^{m+1}$, which preserves $S^{m}$, extends to a complex linear action by $r \in U(m+1)$ on $\mathbb{C}^{m+1}$ which preserves both $A_{1}^{m}$ and $A_{1}^{m} \cap \mathfrak{R e}\left(\mathbb{C}^{m+1}\right)$. Since this action moreover preserves $\eta$, it is a Kähler isometry of $A_{1}^{m}$. Finally, it can be checked that the corresponding action on $T^{*} S^{m}$ induced by the identification $\Psi$ coincides with the canonical symplectomorphism $r^{*}$. In conclusion, using $i$ to denote the almost complex structure on $T^{*} S^{m}$ induced by the above identification $\Psi$, we have shown:

Lemma 3.4 $\left(T^{*} S^{m}, i, d \theta_{S^{m}}\right)$ is a Kähler manifold for which each

$$
r^{*}:\left(T^{*} S^{m}, i, d \theta_{S^{m}}\right) \rightarrow\left(T^{*} S^{m}, i, d \theta_{S^{m}}\right), \quad r \in O(m+1),
$$

is a Kähler isometry.

3.2.2 A tame almost complex structure on $T^{*} S^{\boldsymbol{m}} \times \boldsymbol{P}$ Let $J_{P}$ be a fixed compatible almost complex structure on $(P, d \theta)$. For simplicity we will assume that, outside of a compact set, $(P, d \theta)$ is exact symplectomorphic to half a symplectization, where $J_{P}$ moreover is cylindrical. In particular, the latter condition implies that the Liouville flow $\phi^{t}: P \rightarrow P$ is a biholomorphism outside of some compact set.

For $\epsilon>0$ sufficiently small, there is a tame almost complex structure $J$ on $T^{*} S^{m} \times P$ determined by the requirement that the diffeomorphism

$$
\begin{aligned}
\Phi:\left(T^{*} S^{m} \times P, i \oplus J_{P}\right) & \rightarrow\left(T^{*} S^{m} \times P, J\right), \\
(\boldsymbol{q}, \boldsymbol{p}, x) & \mapsto\left(\boldsymbol{q}, \boldsymbol{p}, \phi^{\epsilon g(\boldsymbol{q})}(x)\right),
\end{aligned}
$$

is $\left(i \oplus J_{P}, J\right)$-holomorphic. It follows that:

Lemma 3.5 The action

$$
\left(r^{*}, \operatorname{id}_{P}\right):\left(T^{*} S^{m} \times P, J\right) \rightarrow\left(T^{*} S^{m} \times P, J\right), \quad r \in O(m) \subset O(m+1),
$$

is $J$-holomorphic and fixes $L$. Moreover, there are holomorphic projections

$$
\begin{aligned}
\tilde{\pi}_{P}:=\pi_{P} \circ \Phi^{-1}:\left(T^{*} S^{m} \times P, J\right) & \rightarrow\left(P, J_{P}\right), \\
\pi_{T^{*} S^{m}}:\left(T^{*} S^{m} \times P, J\right) & \rightarrow\left(T^{*} S^{m}, i\right),
\end{aligned}
$$

where

$$
\begin{aligned}
\pi_{T^{*} S^{m}} & =\pi_{T^{*} S^{m} \circ \Phi^{-1},}, \\
\tilde{\pi}_{P}(L) & =\Pi_{L}(\Lambda) \subset P,
\end{aligned}
$$

and $\pi_{P}: T^{*} S^{m} \times P \rightarrow P$ denotes the canonical projection. 
We let $\pi_{m+1}: \mathbb{C}^{m+1} \rightarrow \mathbb{C}$ denote the holomorphic projection onto the last complex coordinate; this projection induces a Lefschetz fibration

$$
\pi_{m+1}: A_{1}^{m} \rightarrow \mathbb{C}
$$

whose critical points are $N$ and $S$ with the critical values 1 and -1 , respectively. We will write

$$
\pi:=\pi_{m+1} \circ \Psi: T^{*} S^{m} \rightarrow \mathbb{C}
$$

for the induced Lefschetz fibration of $T^{*} S^{m}$. The image of $\left(\pi \circ r^{*} \circ \pi_{T^{*} S^{m}}\right)(L) \subset \mathbb{C}$ for any $r \in O(m)$ is shown schematically in Figure 1.

Finally, the set $\pi_{T^{*} S^{m}}(L)$ is shown in Figure 3 in the case $m=1$.

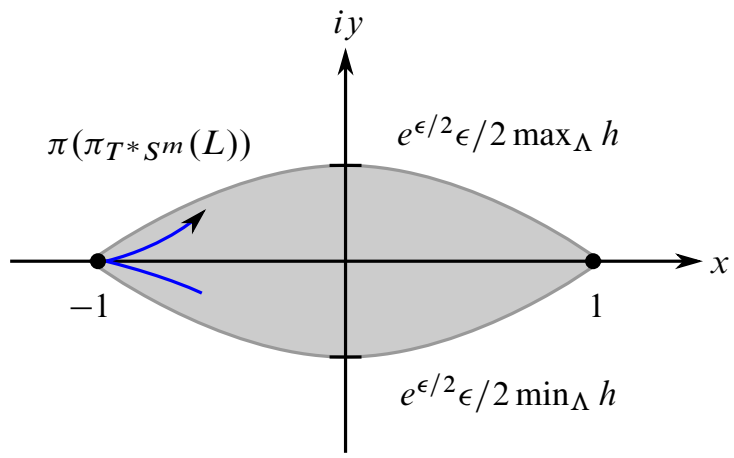

Figure 1: The image of $L \subset T^{*} S^{m} \times P$ under the holomorphic projection $\pi \circ \pi_{T^{*} S^{m}}$, where $\pi: T^{*} S^{m} \rightarrow \mathbb{C}$ is the Lefschetz fibration as described above. Here $\pi(N)=1$ and $\pi(S)=-1$. The arrow schematically depicts the behavior of the boundary near the positive puncture for a $J_{-}$ holomorphic disk having boundary on $L$ and a positive puncture contained inside $\pi_{T^{*} S^{m}}^{-1}(S)$.

\subsection{Transversality results}

Recall that a pseudo-holomorphic disk

$$
u:\left(D^{2}, \partial D^{2}\right) \rightarrow\left(T^{*} S^{m} \times P, L\right)
$$

is said to have a positive (resp. negative) boundary puncture at $p \in \partial D^{2}$ if $u(p)$ is a double point of $L$ and the $z$-coordinate of $L$ jumps to a higher value (resp. lower value) at the puncture when traversing the boundary of the disk according to the orientation. The following lemma is important and relates the transversality of a $J$-holomorphic disk inside $\pi^{-1}(S) \subset T^{*} S^{m} \times P$ having boundary on $L$ with the transversality of its 
projection to $P$, which is a $J_{P}$-holomorphic disk having boundary on $\Pi_{L}(\Lambda)$. Observe that the analogous result for the corresponding disks inside $\pi^{-1}(N) \subset T^{*} S^{m} \times P$ is false in general, which is the reason why computing the full differential $\partial_{L}$ in terms of $\partial_{\Lambda}$ is a more difficult problem.

Lemma 3.6 The $J$-holomorphic disk $u=(S, \tilde{u})$ in $T^{*} S^{m} \times P$ having boundary on $L$ is transversely cut out if and only if the $J_{P}$-holomorphic disk $\tilde{u}$ having boundary on $\Pi_{L}(\Lambda)$ is transversely cut out.

Proof We let $\widetilde{D}_{\tilde{u}}$ and $D_{u}$ denote the linearizations of the Cauchy-Riemann operators $\partial_{J_{P}}$ and $\partial_{J}$ at the solutions $\tilde{u}$ and $u$, respectively. We also linearize the boundary condition and we use $\operatorname{ker} \widetilde{D}_{\tilde{u}}$ and $\operatorname{ker} D_{u}$ to denote the spaces of solutions to the corresponding boundary value problems. From the definition of the index of a Fredholm operator it follows that

$$
\begin{aligned}
& \operatorname{dim} \operatorname{ker} \widetilde{D}_{\tilde{u}} \geq \operatorname{index} \widetilde{D}_{\tilde{u}}, \\
& \operatorname{dim} \operatorname{ker} D_{u} \geq \operatorname{index} D_{u},
\end{aligned}
$$

with equality if and only if the corresponding solution is transversely cut out.

In this case, one checks that the Fredholm indices for the linearized boundary value problems satisfy

$$
\operatorname{index} \widetilde{D}_{\tilde{u}}=\operatorname{index} D_{u} \text {. }
$$

This can be seen by an explicit calculation utilizing Lemma 3.3 and the index formula for the pseudo-holomorphic disks under consideration; see eg [8].

There is a projection

$$
T \tilde{\pi}_{P}: \Gamma\left(u^{*} T\left(T^{*} S^{m} \times P\right)\right) \rightarrow \Gamma\left(u^{*} T P\right)
$$

of sections, induced by the differential of $\tilde{\pi}_{P}$.

The "only if" part is straight-forward: the kernel of $\widetilde{D}_{\tilde{u}}$ lifts to the kernel of $D_{u}$ under $T \tilde{\pi}_{P}$. Hence, if $\tilde{u}$ is not transversely cut out, ie if $\operatorname{dim} \operatorname{ker} \widetilde{D}_{\tilde{u}}>$ index $\widetilde{D}_{\tilde{u}}$, it follows that $\operatorname{dim} \operatorname{ker} D_{u}>$ index $\widetilde{D}_{\tilde{u}}=$ index $D_{u}$ and $u$ is not transversely cut out either.

We proceed to show that $T \tilde{\pi}_{P}$ restricts to an injection

$$
\left.T \tilde{\pi}_{P}\right|_{\text {ker } D_{u}}: \operatorname{ker} D_{u} \rightarrow \operatorname{ker} \widetilde{D}_{\tilde{u}} \text {. }
$$

Observe that the sought statement would follow from the injectivity of $\left.T \tilde{\pi}_{P}\right|_{\operatorname{ker} D_{u}}$. Namely, ker $\widetilde{D}_{\tilde{u}}=$ index $\widetilde{D}_{\tilde{u}}$ implies that

$$
\operatorname{ker} D_{u} \leq \operatorname{ker} \widetilde{D}_{\tilde{u}}=\operatorname{index} \widetilde{D}_{\tilde{u}}=\operatorname{index} D_{u}
$$


and hence that $\operatorname{ker} D_{u}=\operatorname{index} D_{u}$.

The fact that $\left.T \tilde{\pi}_{P}\right|_{\text {ker } D_{u}}$ is an injection is shown as follows (it can be seen as an infinitesimal version of Lemma 3.7). Assume that we are given $\left.\zeta \in \operatorname{ker} T \tilde{\pi}_{P}\right|_{\operatorname{ker} D_{u}}$. The fact that $\zeta$ is in the kernel of $T \tilde{\pi}_{P}$ implies that it can be considered as a holomorphic map from the disk into

$$
\left(T_{S}\left(T^{*} S^{m}\right), i\right) \simeq\left(\mathbb{C}^{m}, i\right) .
$$

We consider the $j^{\text {th }}$ component $\zeta_{j}$ of this map. There is a constant $c>0$ for which the following holds. Recall that $h: \Lambda \rightarrow \mathbb{R}$ is the $z$-coordinate of $\Lambda$. Let $p_{+}$and $p_{-}$, with $h\left(p_{+}\right)>h\left(p_{-}\right)$, be the two lifts of the double point $p \in \Pi_{L}(\Lambda)$ which is the image of the positive puncture of $\tilde{u}$. Further, let

$$
h_{\max }:=c \max _{\Lambda} h, \quad h_{\min }:=c \min _{\Lambda} h, \quad h_{+}:=c h\left(p_{+}\right), \quad h_{-}:=c h\left(p_{-}\right) .
$$

For a suitable choice of holomorphic coordinates and constant $c>0$, the linearized boundary condition implies that $\zeta_{j}$ is contained inside the double cone $C_{+} \cup C_{-}$, where

$$
C_{+}:=\left\{x+i y \mid y \in\left[h_{\min } x, h_{\max } x\right], x \geq 0\right\} \subset \mathbb{C} \quad \text { and } \quad C_{-}:=-C_{+},
$$

as shown in Figure 2. Furthermore, $\zeta_{m}$ necessarily vanishes at the positive puncture, while it is asymptotic to the line

- $y=h_{-} x$ when approaching the positive puncture along the boundary in the direction of the orientation, and

- $y=h_{+} x$ when approaching the positive puncture along the boundary against the direction of the orientation.

The open mapping theorem implies that the interior of $\zeta_{m}$ is mapped to either $C_{+}$ or $C_{-}$. Together with the asymptotics of $\zeta_{m}$ at the positive puncture, the open mapping theorem now shows that $\zeta_{m}$ in fact must vanish identically. Hence $\zeta \equiv 0$, which establishes the injectivity of $\left.T \tilde{\pi}_{P}\right|_{\operatorname{ker} D_{u}}$.

We continue to show that the above lemma can indeed be applied to the $J$-holomorphic disks contributing to $\partial_{L}(s)$ for $s \in \mathcal{Q}_{S}$.

Lemma 3.7 Let $u$ be a $J$-holomorphic disk in $T^{*} S^{m} \times P$ having boundary on $L$ and exactly one positive boundary puncture. If the positive puncture of $u$ is contained in $\pi_{T^{*} S^{m}}^{-1}(S)$, then all of $u$ is contained inside $\pi_{T^{*} S^{m}}^{-1}(S)$. In particular, such disks are in bijective correspondence with the corresponding $J_{P}$-holomorphic disks in $P$ with boundary on $\Lambda$. Finally, given that $J_{P}$ is regular, it follows that these $J$-holomorphic disks are transversely cut out as well. 


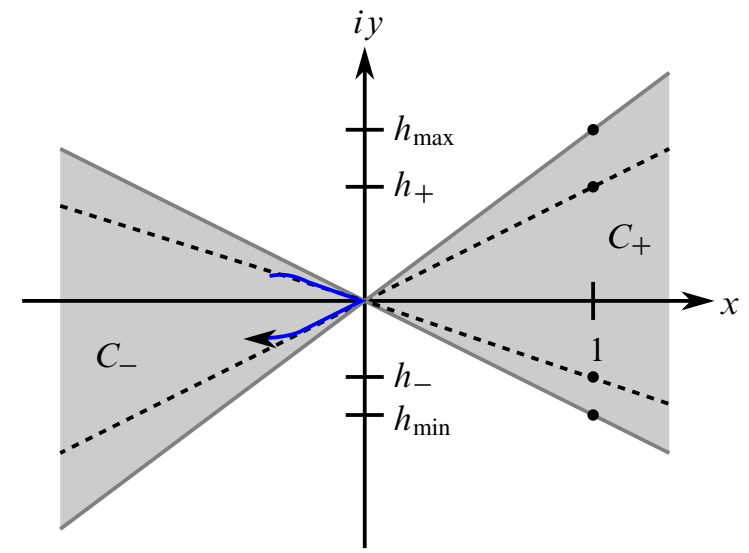

Figure 2: The double cone $C_{-} \cup C_{+}$depicts the projection of the linearized boundary condition near a puncture in $\pi_{T^{*} S^{m}}^{-1}(S)$. The arrow shows the behavior of a solution to the linearized problem along the boundary near the positive puncture, given that it is non-vanishing.

Proof In the case $m=1$, this follows eg from [9, Lemma 4.14]. We here provide a proof of the general case. The idea is to study the image of $\pi_{T^{*} S^{m}} \circ u$ under the Lefschetz fibration $\pi: T^{*} S^{m} \rightarrow \mathbb{C}$. The image of $L$ under this projection is shown in Figure 1.

To that end, we first observe that $\pi \circ \pi_{T^{*} S^{m}} \circ u$ is holomorphic. The asymptotics at the positive puncture (see the arrow in Figure 1 for the projection of the boundary in the case when the projection is non-zero) together with the open mapping theorem implies that $\pi \circ \pi_{T^{*} S^{m}} \circ u$ is constantly equal to $-1 \in \mathbb{C}$ in some neighborhood of

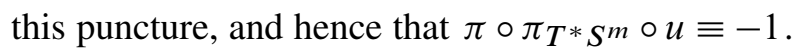

Under the biholomorphism $\Psi$, the fiber $\pi^{-1}(-1) \subset T^{*} S^{m}$ gets identified with the singular quadric

$$
Q:=\left\{z_{1}^{2}+\cdots+z_{m}^{2}=0\right\} \cap A_{1}^{m} \subset \mathbb{C}^{m+1},
$$

such that $S$ is identified with the unique singular point $(0, \ldots, 0,-1) \in Q$. Since $\pi_{T^{*} S^{m}} \circ u$ maps into this fiber and the boundary is mapped to

$$
\pi_{T^{*} S^{m}}(L) \cap \pi^{-1}(-1)=\{S\}
$$

(ie the singular point), the maximum principle now implies that $\pi_{T^{*} S^{m}} \circ u \equiv S$. In other words, $u$ is contained in $\pi_{T^{*} S^{m}}^{-1}(S)$.

Conversely, for each $J_{P}$ holomorphic disk $\tilde{u}$ in $P$ having boundary on $\Pi_{L}(\Lambda)$ one immediately constructs a $J$-holomorphic disk $u=(S, \tilde{u})$ in $T^{*} S^{m} \times P$ having boundary on $L$. 
The transversality statement follows from Lemma 3.6 above.

Lemma 3.8 A non-constant $J$-holomorphic disk $u$ in $T^{*} S^{m} \times P$ with boundary on $L$ and exactly one positive puncture contained in $\pi_{T^{*} S^{m}}^{-1}(N)$ has index

$$
\operatorname{index}(u)=\operatorname{index}\left(\tilde{\pi}_{P} \circ u\right)+m-N_{u} m,
$$

where $N_{u}$ is the number of negative punctures of $u$ contained in $\pi_{T^{*} S^{m}}^{-1}(N)$.

If $\tilde{\pi}_{P} \circ u$ is non-constant, under the additional assumption that $J_{P}$ is a regular almost complex structure it follows that

$$
\operatorname{index}(u) \geq m-N_{u} m \text {. }
$$

If $\tilde{\pi}_{P} \circ u \equiv p \in P$ is constant, it follows that $u$ is a pseudo-holomorphic strip whose negative puncture is contained in $\pi_{T^{*} S^{m}}^{-1}(S)$, both of whose punctures correspond to $p \in \mathcal{Q}(\Lambda)$ and whose index satisfies

$$
\operatorname{index}(u)=m-1 \geq 0 .
$$

In the case $m=1$ this strip is, moreover, transversely cut out.

Proof Formula (3-1) follows from a simple index calculation utilizing Lemma 3.3 and the index formula in [8].

Suppose that $\tilde{\pi}_{P} \circ u$ is not constant. The inequality (3-2) follows from (3-1) together with the inequality index $\left(\tilde{\pi}_{P} \circ u\right) \geq 0$. To see the latter inequality, recall that the projection

$$
\tilde{\pi}_{P}:\left(T^{*} S^{m} \times P, L\right) \rightarrow\left(P, \Pi_{L}(\Lambda)\right)
$$

is $\left(J, J_{P}\right)$-holomorphic by Lemma 3.5 , and that $J_{P}$ is regular by assumption, and hence that the index of the solution is equal to the dimension of the moduli space in which it is contained.

Suppose that $\tilde{\pi}_{P} \circ u$ is constant. First, it follows that we have $u=(\tilde{u}, p)$, where $p \in \Pi_{L}(\Lambda)$ is a double point and $\tilde{u}: D^{2} \rightarrow T^{*} S^{m}$ is holomorphic. It follows that $u$ must be a strip whose positive puncture (resp. negative puncture) is the puncture in $\mathcal{Q}_{N}$ (resp. $\mathcal{Q}_{S}$ ) corresponding to $p$. From this fact we get (3-3).

Finally, the transversality claim in the case $m=1$ follows by an explicit calculation that is standard. To that end, observe that the strip $\tilde{u}$ is an embedding of a shaded strip, as shown in Figure 3. 
Recall that a broken $J$-holomorphic disk consists of a connected directed tree satisfying the following conditions. Let $V$ and $E$ denote the vertex and edge sets of the tree, respectively. First, each vertex $v \in V$ is assigned a $J$-holomorphic disk $u_{v}$ with exactly one positive puncture. Second, each edge $e \in E$, also called a node of the broken configuration, is assigned a negative puncture $q_{e}$ of $u_{v}$ and a positive puncture $p_{e}$ of $u_{w}$, where $v$ and $w$ are the starting point and endpoint of $e$, respectively, and for which $u_{v}\left(q_{e}\right)=u_{w}\left(p_{e}\right)$ is required to hold. By a positive (respectively, negative) puncture of a broken $J$-holomorphic disk we mean a positive (respectively, negative) puncture of one of the involved disks which does not correspond to any node.

Lemma 3.9 Assume that $J_{P}$ is regular. A broken $J$-holomorphic disk $u$ in $T^{*} S^{m} \times P$ with boundary on $L$, exactly one positive puncture contained in $\pi_{T^{*} S^{m}}^{-1}(N)$, and all of its negative punctures contained in $\pi_{T^{*} S^{m}}^{-1}(S)$, must be of positive index.

Proof Suppose that the broken disk consists of the $J$-holomorphic discs $\left\{u_{i}\right\}_{i=1}^{v+1}$ and $\left\{v_{i}\right\}_{i=1}^{\mu}$, where $u_{i}$ has its positive puncture contained in $\pi_{T^{*} S^{m}}^{-1}(N)$ and $v_{i}$ has its positive puncture contained in $\pi_{T^{*} S^{m}}^{-1}(S)$. Furthermore, we may order the disks $\left\{u_{i}\right\}$ so that $\tilde{\pi}_{P} \circ u_{i}$ is constant if and only if $k<i \leq v+1$ for some $0 \leq k \leq v+1$. Observe that $\mu+v$ is the total number of nodes of the broken disk.

The index of the broken disk is computed to be

$$
I:=v+\sum_{i=1}^{v+1} \operatorname{index}\left(u_{i}\right)+\mu+\sum_{i=1}^{\mu} \operatorname{index}\left(v_{i}\right),
$$

where Lemma 3.7 implies that $\mu+\sum_{i=1}^{\mu} \operatorname{index}\left(v_{i}\right) \geq 0$.

In the case $k=0$ Lemma 3.8 implies that $v=0$ and, since $\mu+v>0$ by assumption, it follows that $\mu>0$. In this case, (3-3) implies that

$$
I \geq \mu+\operatorname{index}\left(u_{0}\right)=\mu+(m-1)>0 .
$$

In the case $k>0,(3-2)$ and (3-3) show that

$$
\begin{aligned}
I \geq v+\sum_{i=1}^{v+1} \operatorname{index}\left(u_{i}\right) & \geq v+\sum_{i=1}^{k}\left(m-m N_{u_{i}}\right)+(v+1-k)(m-1) \\
& =v+m\left(k-\sum_{i=1}^{k} N_{u_{i}}\right)+(v+1-k)(m-1) .
\end{aligned}
$$

Finally, the assumption that all negative punctures of the broken disk are contained in $\pi_{T^{*} S^{m}}^{-1}(S)$ together with the fact that the disks $v_{i}$ have all negative punctures contained 
in $\pi_{T^{*} S^{m}}^{-1}(S)$ by Lemma 3.7 gives the inequality $v \geq \sum_{i=1}^{k} N_{u_{i}}$. In conclusion, we have

$$
v+\sum_{i=1}^{v+1} \operatorname{index}\left(u_{i}\right) \geq v+m(k-v)+(v+1-k)(m-1)=m-1+k>0 .
$$

\subsection{A sign computation in the case $m=1$}

Lemma 3.10 Let $u$ be a $J$-holomorphic disk in $T^{*} S^{1} \times P$ having boundary on $L$. If the projection $\pi_{T^{*} S^{1}} \circ u$ is non-constant, then the image of $\pi_{T^{*} S^{1}} \circ u$ intersects the interior of exactly one of the two subsets

$$
\begin{gathered}
T^{*}\left\{(\cos \theta, \sin \theta) \mid \frac{1}{2} \pi \leq \theta \leq \frac{3}{2} \pi\right\} \subset T^{*} S^{1}, \\
T^{*}\left\{(\cos \theta, \sin \theta) \mid-\frac{1}{2} \pi \leq \theta \leq \frac{1}{2} \pi\right\} \subset T^{*} S^{1} .
\end{gathered}
$$

Proof By Lemma 3.7, we may suppose that the positive puncture of the disk is contained in $\pi_{T^{*} S^{1}}^{-1}(N)$, since it would otherwise have a constant projection to $S \in T^{*} S^{1}$.

Further, unless its projection to $T^{*} S^{1}$ is constant, an interior point of the disk cannot be mapped into $\pi_{T^{*} S^{1}}^{-1}\{S \cup N\}$, as follows from the open mapping theorem applied to the holomorphic projection $\pi_{T^{*} S^{1}} \circ u$. To that end, we refer to Figure 3 for a description of the image of the boundary condition $\pi_{T^{*} S^{1}}(L)$ under this projection.

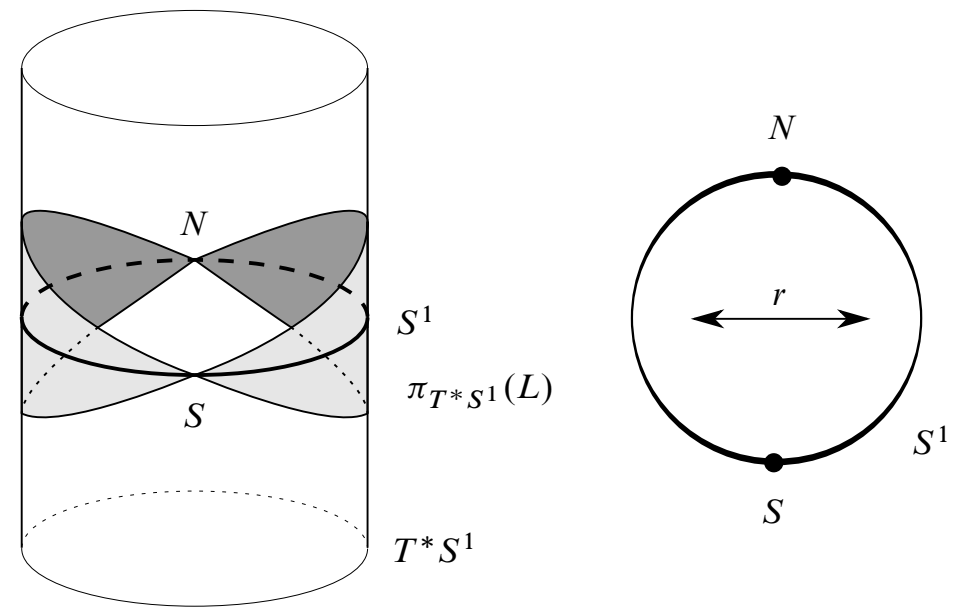

Figure 3: The image of $L \subset P \times T^{*} S^{1}$ under the projection $\pi_{T^{*} S^{1}}$. Observe that $\pi_{T^{*} S^{1}}(L)$ is invariant under $r^{*}$, where $r \in O(1)$. 
Lemma 3.11 The $J$-holomorphic involution $\left(r^{*}, \mathrm{id}_{P}\right)$ acts non-trivially on the $J-$ holomorphic disks in $T^{*} S^{1} \times P$ having boundary on $L$ and non-constant projection to $T^{*} S^{1}$.

In the case when $\Lambda$ is spin and the $J$-holomorphic disk $u=(\tilde{u}, p), p \in P$, is a nontrivial strip in $T^{*} S^{1} \times P$ with boundary on $L$, the involution applied to $u$ moreover reverses the coherent orientation.

Proof The first claim follows immediately from Lemma 3.10.

To check the effect on the coherent orientation of the moduli space containing a $J_{-}$ holomorphic strip $u$ as in the claim, we argue as follows. By Lemma 3.10 it follows that $u$ and $r^{*} \circ u$ have homotopic boundaries after "capping off" the boundary with a choice of capping path at each puncture. The claim follows since the involution $\left(r^{*}, \mathrm{id}_{P}\right)$ restricts to an orientation-reversing involution on $L$ and since it does not fix $u$.

\subsection{Proof of Theorem 3.1}

We choose the perturbation $L$ of $S^{m} \times \Lambda$ as constructed in Section 3.1, for which we have the decomposition $\mathcal{Q}(L)=\mathcal{Q}_{N} \sqcup \mathcal{Q}_{S}$ of the Reeb chords. The grading-preserving bijection between the Reeb chords $\mathcal{Q}_{S}$ on $L$ and the Reeb chords on $\Lambda$, as established in Lemma 3.3, thus induces a graded unital algebra inclusion

$$
\iota: \mathcal{A}_{\bullet}(\Lambda) \hookrightarrow \mathcal{A}_{\bullet}(L) .
$$

Perturb the almost complex structure $J$ constructed in Section 3.2 to a regular, almost complex structure $J^{\prime}$. For a sufficiently small such perturbation, Lemma 3.7 implies that $\iota$ is a chain map. Here we have used the fact that the number of solutions in a transversely cut-out rigid moduli space stays the same after a sufficiently small such perturbation.

Observe that $\iota$ has a left inverse

$$
\pi: \mathcal{A}_{\bullet}(L) \rightarrow \mathcal{A}_{\bullet}(L) /\left\langle\mathcal{Q}_{N}\right\rangle=\mathcal{A}_{\bullet}(\Lambda)
$$

on the algebra level, which is induced by the quotient with the two-sided ideal generated by $\mathcal{Q}_{N}$. We will show that, given that $J^{\prime}$ is sufficiently close to $J, \pi$ is in fact a chain map, from which the theorem follows.

The fact that $\pi$ is a chain map is equivalent to $\partial_{L}$ preserving the two-sided ideal $\left\langle\mathcal{Q}_{N}\right\rangle$. To that end, for each $c \in \mathcal{Q}_{N}$, we need to show that $\partial_{L}(c)$ may be assumed to consist of a sum of words all which contain at least one letter from $\mathcal{Q}_{N}$. 
Taking the limit $J^{\prime} \rightarrow J$, the solutions of $J^{\prime}$-holomorphic disks converge to (possibly broken) $J$-holomorphic disks by the Gromov-Floer-type compactness used in [10]. In particular, if the set of (possibly broken) solutions of $J$-holomorphic disks is empty for some specifications of the punctures, then the same is true for the corresponding moduli space of $J^{\prime}$-holomorphic disks given that $J^{\prime}$ is a sufficiently small perturbation of $J$. Lemma 3.9 implies that the set of broken $J$-holomorphic disks of index zero which (after perturbing $J$ ) could contribute to a term in the expression $\partial_{L}(c)$ all have at least one negative puncture in $\mathcal{Q}_{N}$. In other words, the $J^{\prime}$-holomorphic disks that contribute to words in the expression $\partial_{L}(c)$ containing no letters from $\mathcal{Q}_{N}$ correspond to unbroken $J$-holomorphic disks.

Case $\boldsymbol{m} \geq \mathbf{2}$ Lemma 3.8 implies that there are no such unbroken disks of index zero, which shows the claim.

Case $\boldsymbol{m}=\mathbf{1}$ Lemma 3.8 implies that such disks of index zero are necessarily $J_{-}$ holomorphic strips that are constant when projected to $P$. By Lemma 3.11, there are exactly two such strips, which, moreover, are transversely cut-out solutions equipped with opposite coherent orientations. In other words, they do not contribute to $\partial(c)$.

\section{Examples beyond augmentable Legendrians}

Recall that for Legendrian submanifolds whose Chekanov-Eliashberg algebra admits an augmentation, (1-1) was proven by Ekholm, Etnyre and Sullivan [9]. Ekholm, Etnyre and Sabloff [7] later improved it to (1-2) under the same assumptions. In addition, given that $\Lambda$ is spin and has a Chekanov-Eliashberg algebra admitting an augmentation into $R=\mathbb{Z}, \mathbb{Q}, \mathbb{R}$ or $\mathbb{Z}_{m}$, we may take $b_{i}:=\operatorname{rk} H_{i}(\Lambda ; R)$ in the inequality.

There are certain examples of Legendrian knots in $\mathbb{C} \times \mathbb{R}$ due to Sivek [29], which will be discussed below, whose characteristic algebras admit 2-dimensional representations over $\mathbb{Z}_{2}$, but no 1 -dimensional representations over $\mathbb{Z}_{2}$. We will use these examples to construct Legendrian submanifolds $\Lambda \subset \mathbb{C}^{n} \times \mathbb{R}$ having Chekanov-Eliashberg algebras that do not admit augmentations in any unital commutative ring $R$, but for which there exists a finite-dimensional representation $\rho: \mathcal{C}_{\Lambda} \rightarrow \mathrm{M}_{k}\left(\mathbb{Z}_{2}\right)$ for some $k>1$.

Recall that the Kauffman bound

$$
\operatorname{tb}(\Lambda) \leq \min -\operatorname{deg}_{a} F_{\Lambda}(a, x)-1
$$

is satisfied for any Legendrian knot $\Lambda \subset \mathbb{C} \times \mathbb{R}$, as shown by Rudolf [26], where $F_{\Lambda}(a, x)$ is the so-called Kauffman polynomial for the underlying smooth knot of $\Lambda$. We will need the following strong condition for the existence of an augmentation. 
Proposition 4.1 (Rutherford [27]; Henry and Rutherford [19]) Let $\mathbb{F}$ be an arbitrary field and $\Lambda \subset \mathbb{C} \times \mathbb{R}$ a Legendrian knot. The Kauffman bound

$$
\operatorname{tb}(\Lambda) \leq \min -\operatorname{deg}_{a} F_{\Lambda}(a, x)-1
$$

is an equality if and only if the Chekanov-Eliashberg algebra of $\Lambda$ defined with Novikov coefficients $\mathbb{F}\left[H_{1}(\Lambda)\right]$ admits an ungraded augmentation into $\mathbb{F}$.

Proof The proof is similar to the proof of [29, Proposition 3.2], which considers the case $\mathbb{F}=\mathbb{Z}_{2}$. The Legendrian knot admits an ungraded ruling if and only if the Kauffman bound is an equality, by [27, Theorem 3.1], and the existence of an ungraded ruling is equivalent to the existence of an ungraded augmentation in $\mathbb{F}$ by [19, Theorem 3.4] (given that the Chekanov-Eliashberg algebra is defined with Novikov coefficients). See [22] for an alternative proof of the latter fact.

Remark 4.2 Regarding higher-dimensional representations of the characteristic algebra, the following can be said. If the characteristic algebra $\mathcal{C}_{\Lambda}$ of a Legendrian knot $\Lambda \subset \mathbb{C} \times \mathbb{R}$ admits a finite-dimensional representation over $\mathbb{Z}_{2}$, then $\Lambda$ maximizes the Thurston-Bennequin invariant within its topological knot type [25, Theorem 1.2]. Furthermore, the existence of such a representation depends only on $\operatorname{tb}(\Lambda)$ and the topological type of $\Lambda$.

We first prove the following lemma, which shows how finite-dimensional representations of characteristic algebras behave under the operation of connected sum, as defined by Etnyre and Honda [16].

Lemma 4.3 Let $\Lambda_{1}$ an $\Lambda_{2}$ be two Legendrian knots in $\mathbb{C} \times \mathbb{R}$.

(1) If the characteristic algebra $\mathcal{C}_{\Lambda_{i}}$ admits a $k_{i}$-dimensional (graded) representation over a field $\mathbb{F}$ for $k_{i} \geq 1$ and $i=1,2$, then $\mathcal{C}_{\Lambda_{1} \# \Lambda_{2}}$ admits a $k_{1} k_{2}$-dimensional (graded) representation over $\mathbb{F}$.

(2) If one of $\Lambda_{i}, i=1,2$, satisfies the strict inequality

$$
\operatorname{tb}\left(\Lambda_{i}\right)<\min -\operatorname{deg}_{a} F_{\Lambda_{i}}(a, x)-1
$$

for the Kauffman bound, then the Chekanov-Eliashberg algebra of $\Lambda_{1} \# \Lambda_{2}$ does not admit an augmentation into any unital commutative ring $R$.

Proof We first prove (1). Observe that there is an exact Lagrangian cobordism $L$ from $\Lambda_{1} \sqcup \Lambda_{2}$ to $\Lambda_{1} \# \Lambda_{2}$ by eg [5] or [11]. It follows that there exists a unital 
algebra morphism $f_{L}: \mathcal{C}_{\Lambda_{1} \# \Lambda_{2}} \rightarrow \mathcal{C}_{\Lambda_{1}} \otimes \mathcal{C}_{\Lambda_{2}}$. If $\rho_{i}: \mathcal{C}_{\Lambda_{i}} \rightarrow \mathrm{M}_{k_{i}}$ (F) is a (graded) $k_{i}-$ dimensional representation of $\mathcal{C}_{\Lambda_{i}}$, then the pullback of the tensor product of (graded) representations $\rho_{1}$ and $\rho_{2}$

$$
\left(f_{L}\right)^{*}\left(\rho_{1} \otimes \rho_{2}\right): \mathcal{C}_{\Lambda_{1} \# \Lambda_{2}} \rightarrow \mathrm{M}_{k_{1} k_{2}}(\mathbb{F})
$$

is a (graded) $k_{1} k_{2}$-dimensional representation of $\mathcal{C}_{\Lambda_{1} \# \Lambda_{2}}$.

We then prove (2). First, observe that for any unital commutative ring $R$ there is a maximal ideal $m \subset R$. Composing the augmentation to $R$ with the quotient projection $R \rightarrow R / m$ thus induces an augmentation into the field $R / m$. It thus suffices to show the statement when $R=\mathbb{F}$ is a field.

Without loss of generality, we assume that the Kauffman bound for $\Lambda_{1}$ is not an equality, ie that

$$
\operatorname{tb}\left(\Lambda_{1}\right)<\min -\operatorname{deg}_{a} F_{\Lambda_{1}}(a, x)-1 .
$$

In addition, $\operatorname{tb}\left(\Lambda_{1} \# \Lambda_{2}\right)=\operatorname{tb}\left(\Lambda_{1}\right)+\operatorname{tb}\left(\Lambda_{2}\right)+1$ [16, Lemma 3.3]. Hence, using the Kauffman bounds for $\operatorname{tb}\left(\Lambda_{1}\right)$ and $\operatorname{tb}\left(\Lambda_{2}\right)$, we get that

$$
\begin{aligned}
\operatorname{tb}\left(\Lambda_{1} \# \Lambda_{2}\right) & =\operatorname{tb}\left(\Lambda_{1}\right)+\operatorname{tb}\left(\Lambda_{2}\right)+1 \\
& <\min -\operatorname{deg}_{a} F_{\Lambda_{1}}(a, x)+\min -\operatorname{deg}_{a} F_{\Lambda_{2}}(a, x)-1 .
\end{aligned}
$$

Further, as shown in [20], we have the equality

$$
\min -\operatorname{deg}_{a} F_{\Lambda_{1} \# \Lambda_{2}}(a, x)=\min -\operatorname{deg}_{a} F_{\Lambda_{1}}(a, x)+\min -\operatorname{deg}_{a} F_{\Lambda_{2}}(a, x),
$$

and hence we see that the Kauffman bound

$$
\operatorname{tb}\left(\Lambda_{1} \# \Lambda_{2}\right)<\min -\operatorname{deg}_{a} F_{\Lambda_{1} \# \Lambda_{2}}(a, x)-1
$$

for $\Lambda_{1} \# \Lambda_{2}$ is not an equality either. Consequently, Proposition 4.1 shows that the Chekanov-Eliashberg algebra of $\Lambda_{1} \# \Lambda_{2}$ defined using Novikov coefficients does not admit an augmentation into $\mathbb{F}$. In particular, it follows that its Chekanov-Eliashberg algebra defined without Novikov coefficients does not admit an augmentation either.

Example 4.4 Sivek [29] considered a Legendrian knot $\Lambda_{p,-q}$, which is a Legendrian representative of $(p,-q)$-torus knot with $p \geq 3$ odd and $q>p$. The front projection of $\Lambda_{5,-8}$ is shown in Figure 4. Sivek proved that the characteristic algebra $\mathcal{C}_{\Lambda_{p,-q}}$ admits a representation $\rho: \mathcal{C}_{\Lambda_{p,-q}} \rightarrow \mathrm{M}_{k}\left(\mathbb{Z}_{2}\right)$ for $k=2$ but not for $k=1$; see [29]. In addition, observe that the proof that $\mathcal{C}_{\Lambda_{p, q}}$ does not admit a 1-dimensional representation over $\mathbb{Z}_{2}$ is based on the fact that the Kauffman bound of $\Lambda_{p,-q}$ is a strict inequality $-p q=\operatorname{tb}\left(\Lambda_{p,-q}\right)<-p q+q-p$; see [15; 29]. Hence, using the same argument as 


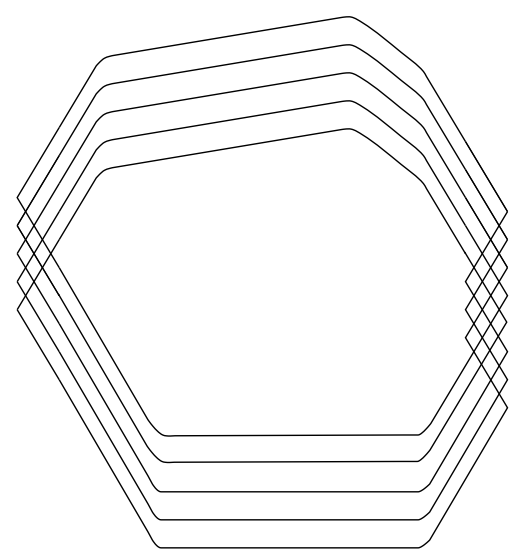

Figure 4: The front projection of $\Lambda_{5,-8}$

in the proof of Lemma 4.3, one gets that $\mathcal{C}_{\Lambda_{p,-q}}$ does not admit an augmentation into a unital commutative ring. Observe that $r\left(\Lambda_{p,-q}\right)=q-p$ (if the orientation is such that the cusps in the left part of Figure 4 are downward cusps). Since we will use it in the proof of Theorem 1.9 below, observe that the Maslov class of $\Lambda_{p,-q}$ is given by $\mu\left(\Lambda_{p,-q}\right)=2(q-p)$.

We now discuss another family of examples.

Example 4.5 Observe that from Example 4.4 and Lemma 4.3 we can get many other examples of Legendrian knots $\Lambda$ whose characteristic algebras admit finitedimensional representations over $\mathbb{Z}_{2}$, but whose Chekanov-Eliashberg algebras do not admit augmentations in any unital commutative ring $R$. Say, consider $\Lambda_{p,-q} \# T_{2, k}$, where $T_{2, k}$ is a Legendrian representative of torus $(2, k)$-knot - see Figure 5 - where

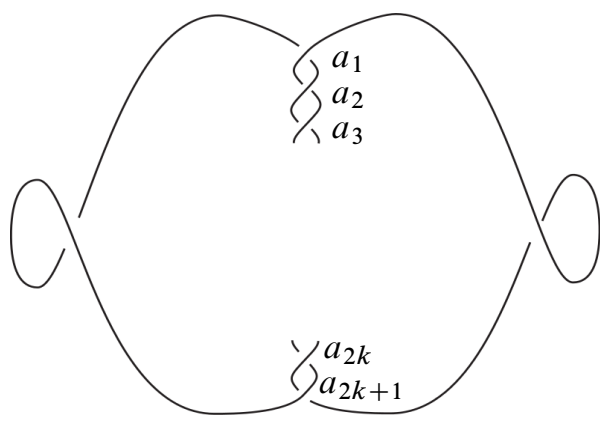

Figure 5: The Lagrangian projection of $T_{2, k}$ 
$p \geq 3$ is odd and fixed, $k \in \mathbb{N}$ is fixed and $q>p$. Since the Chekanov-Eliashberg algebra of $T_{2, k}$ admits an augmentation into $\mathbb{Z}_{2}$ - see [11] - its characteristic algebra has a 1 -dimensional representation over $\mathbb{Z}_{2}$. Then we apply Lemma 4.3 and see that $\Lambda_{p,-q} \# T_{2, k}$ satisfies the sought properties. In addition, since $r\left(T_{2, k}\right)=0$, observe that $r\left(\Lambda_{p,-q} \# T_{2, k}\right)=q-p$ and one can find infinitely many such knots which are pairwise not Legendrian isotopic. We also note that the Maslov class $\mu\left(\Lambda_{p,-q} \# T_{2, k}\right)$ equals $2(q-p)$.

\subsection{Proof of Theorem 1.9}

Consider a family of Legendrian knots $\left(\tilde{\Lambda}_{l}\right)_{l \in \mathbb{N}}$ satisfying the following three properties:

(1) $\mu\left(\tilde{\Lambda}_{l_{1}}\right) \neq \mu\left(\tilde{\Lambda}_{l_{2}}\right)$ whenever $l_{1} \neq l_{2}$ in $\mathbb{N}$.

(2) $\mathcal{A}\left(\tilde{\Lambda}_{l}\right)$ does not admit an augmentation to any unital commutative ring $R$.

(3) $\mathcal{C}_{\tilde{\Lambda}_{l}}$ admits a finite-dimensional representation $\rho_{l}: \mathcal{C}_{\tilde{\Lambda}_{l}} \rightarrow \mathrm{M}_{k}\left(\mathbb{Z}_{2}\right), k>1$.

We can use a family $\tilde{\Lambda}_{l}:=\Lambda_{p,-p-l}$, described in Example 4.4, with fixed $p \geq 3$ odd and $l \in \mathbb{N}$, or a family $\widetilde{\Lambda}_{l}:=\Lambda_{p,-p-l} \# T_{2, k}$, described in Example 4.5, where $l \in \mathbb{N}$ and we have fixed $p \geq 3$ odd and $k \in \mathbb{N}$. We also observe that any family of Legendrian knots $\left(\Lambda_{k}^{\prime}\right)_{k \in \mathbb{N}}$ such that $\mathcal{A}\left(\Lambda_{k}^{\prime}\right)$ admits an augmentation in $\mathbb{Z}_{2}$ and $\mu\left(\Lambda_{k_{1}}^{\prime}\right)=\mu\left(\Lambda_{k_{2}}^{\prime}\right)$ for all $k_{1}, k_{2} \in \mathbb{N}$ will lead to a family $\left(\Lambda_{l}\right)_{l \in \mathbb{N}}$, where $\tilde{\Lambda}_{l}:=\Lambda_{p,-p-l} \# \Lambda_{k}^{\prime}$, which satisfies the sought properties.

We now define $\Lambda_{l}:=\Sigma_{S^{m_{s}}} \cdots \Sigma_{S^{m_{1}}} \widetilde{\Lambda}_{l}$. Observe that, from property (1) and the result of Lambert-Cole [21], it follows that $\mu\left(\Lambda_{l_{1}}\right) \neq \mu\left(\Lambda_{l_{2}}\right)$ for all $l_{1} \neq l_{2}$ in $\mathbb{N}$. Therefore, the $\Lambda_{l}$ are pairwise not Legendrian isotopic. From the first two parts of Theorem 1.8 and properties (2) and (3) it follows that $\mathcal{C}_{\Lambda_{l}}$ admits a finite-dimensional representation $\rho_{l}: \mathcal{C}_{\Lambda_{l}} \rightarrow \mathrm{M}_{k}\left(\mathbb{Z}_{2}\right), k>1$, but that $\mathcal{A}\left(\Lambda_{l}\right)$ does not admit an augmentation to any unital commutative ring $R$. Finally, we see that Theorem 1.6 is applicable to $\Lambda_{l}$. This finishes the proof.

\section{Limitations of the argument}

Note that our proof of Theorem 1.6 is based on the fact that if $\mathcal{C}_{\Lambda}$ admits a $k$ dimensional representation $\rho$, then we can reduce the $\mathcal{C}_{\Lambda}-\mathcal{C}_{\Lambda^{\prime}}$-bimodule $\mathcal{C}\left(\Lambda, \Lambda^{\prime}\right)$ to the $\mathrm{M}_{k}(\mathbb{F})-\mathrm{M}_{k}(\mathbb{F})$-bimodule $\mathcal{C}_{\rho}\left(\Lambda, \Lambda^{\prime}\right)$, which leads to rank inequality (2-1) (which in the simplest situation of a short exact sequence of finitely generated abelian groups is equivalent to an application of the rank-nullity theorem). In order to have this type of 


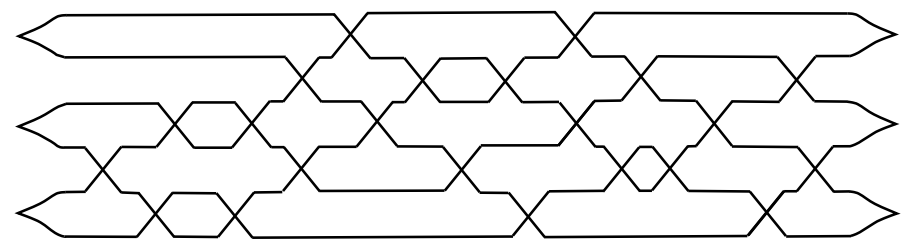

Figure 6: The front projection of $\Lambda_{m\left(10_{132}\right)}$ defined by the braid words $4,5,3,5,3,2,4,1,3,2,4,2,5,1,3,2,4,4,3,5,4,2$; cf [29, Figure 2]

inequality for ranks of bimodules, the minimal imaginable requirement on $\mathcal{C}_{\Lambda} \otimes \mathcal{C}_{\Lambda^{\prime}}^{\text {op }}$ is to have a well-defined notion of rank for free left $\mathcal{C}_{\Lambda} \otimes \mathcal{C}_{\Lambda^{\prime}}^{\mathrm{op}}$-modules (the so-called IBN property) which, moreover, satisfies the property that the rank of a free module cannot be exceeded by the rank of a free submodule (the so-called strong rank property).

Remark 5.1 Assume that we are given a Legendrian submanifold $\Lambda \subset P \times \mathbb{R}$ for which $\mathcal{C}_{\Lambda}$ does not satisfy the rank property. From the fact that there exists a natural unital homomorphism $\mathcal{C}_{\Lambda} \rightarrow \mathcal{C}_{\Lambda} \otimes \mathcal{C}_{\Lambda^{\prime}}^{\text {op }}$ and Remark 1.3 it follows that $\mathcal{C}_{\Lambda} \otimes \mathcal{C}_{\Lambda^{\prime}}^{\text {op }}$ does not satisfy the rank property either. Hence, using Remark 1.4 it follows that $\mathcal{C}_{\Lambda} \otimes \mathcal{C}_{\Lambda^{\prime}}^{\text {op }}$ in particular does not satisfy the strong rank property.

We now proceed to prove the following simple fact:

Fact 5.2 If $R$ is a unital ring for which there exist elements $x, y, p, q \in R$ satisfying

$$
p(1-y x) q=1 \quad \text { and } \quad x y=1,
$$

then $R$ does not satisfy the rank property.

Proof Consider a column vector $(x, p(1-y x))^{T}$ and a row vector $(y,(1-y x) q)$. It is easy to see that

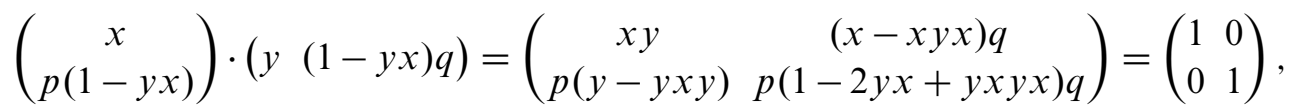

and hence using Remark 1.5 we get that $R$ does not satisfy the rank property.

We now discuss an example of a Legendrian knot $\Lambda \subset \mathbb{C} \times \mathbb{R}$ for which $\mathcal{C}_{\Lambda}$ does not satisfy the rank property.

Consider the Legendrian representative $\Lambda_{m\left(10_{132}\right)}$ of $m\left(10_{132}\right)$ shown in Figure 6. We recall some facts about its characteristic algebra $\mathcal{C}_{\Lambda_{m\left(10_{132}\right)}}=\mathbb{Z}_{2}\left\langle x_{1}, \ldots, x_{25}\right\rangle / B$, using the notation of [29], where $B$ is a two-sided ideal generated by $\left\{\partial x_{1}, \ldots, \partial x_{25}\right\}$. Setting $x:=1+x_{5}\left(x_{2}+x_{3}\right)$ and $y:=x_{20}$, Sivek [29] showed that $x y=1$ and that 
the two-sided ideal $I \subset \mathcal{C}_{\Lambda_{m\left(10_{132}\right)}}$ generated by $1-y x$ coincides with $\mathcal{C}_{\Lambda_{m\left(10_{132}\right)} \text {. }}$. Even more can be said: taking $p:=x_{13}+x_{8}\left(x_{2}+x_{3}\right)$ and $q:=x_{18}$, we moreover have $p(1-y x) q=1$. This follows from the formulas

$$
\partial x_{22}=x q \quad \text { and } \quad \partial x_{23}=1+x_{11} x_{22}+p q
$$

in [29], taking into count that $x_{11}$ vanishes in $\mathcal{C}_{\Lambda_{m\left(10_{132}\right)}}$ (as computed in [29, Section 2.2]). Hence, using Fact 5.2, we see that $\mathcal{C}_{\Lambda_{m\left(10_{132}\right)}}$ does not satisfy the rank property.

Note that it follows from Remark 5.1 that $\mathcal{C}_{\Lambda} \otimes \mathcal{C}_{\Lambda^{\prime}}^{\text {op }}$ does not satisfy the strong rank property for $\Lambda=\Lambda_{m\left(10_{132}\right)}$ either.

Proof of Theorem 1.10 Assume that we are given a Legendrian knot $\widetilde{\Lambda} \subset \mathbb{C} \times \mathbb{R}$ for which $\mathcal{C}_{\tilde{\Lambda}}$ does not satisfy the rank property. For example, $\tilde{\Lambda}=\Lambda_{m\left(10_{132}\right)}$.

We then use the spherical front spinning construction. For a given $n \in \mathbb{N}$, consider $\Sigma_{S^{m_{s}}} \cdots \Sigma_{S^{m_{1}}} \tilde{\Lambda}$, where $\sum_{i=1}^{s} m_{i}+1=n$. Observe that from Theorem 3.1 it follows

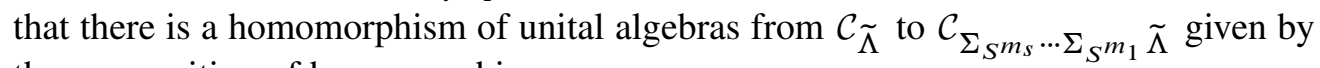
the composition of homomorphisms

$$
\mathcal{C}_{\tilde{\Lambda}} \rightarrow \mathcal{C}_{\Sigma_{S^{m_{1}}} \tilde{\Lambda}} \rightarrow \cdots \rightarrow \mathcal{C}_{\Sigma_{S^{m_{s}}} \ldots \Sigma_{S^{m_{1}}} \tilde{\Lambda}}
$$

Since $\mathcal{C}_{\tilde{\Lambda}}$ does not satisfy the rank property, it follows from Remark 1.3 that neither

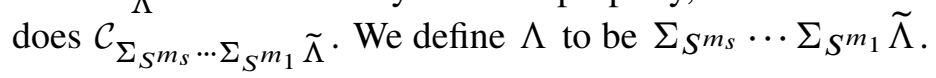

We also observe that Remark 5.1 implies that $\mathcal{C}_{\Lambda} \otimes \mathcal{C}_{\Lambda^{\prime}}^{\text {op }}$ does not satisfy the strong rank property. This finishes the proof.

Remark 5.3 If there exists an exact Lagrangian cobordism $L$ from $\Lambda_{-}$to $\Lambda_{+}$and $\mathcal{C}_{\Lambda_{+}}$does not satisfy the rank property, then the same is true for $\mathcal{C}_{\Lambda_{-}}$. Recall that $L$ induces a unital DGA-morphism $\mathcal{A}\left(\Lambda_{+}\right) \rightarrow \mathcal{A}\left(\Lambda_{-}\right)$and hence a unital algebra morphism $\mathcal{C}_{\Lambda_{+}} \rightarrow \mathcal{C}_{\Lambda_{-}}$. Remark 1.3 now implies the claim.

Remark 5.4 Assume that we are given a Legendrian submanifold $\Lambda \subset P \times \mathbb{R}$ for which $\mathcal{C}_{\Lambda}$ does not satisfy the rank property. It follows from Remark 1.3 that, even if we try to reduce $\mathcal{C}\left(\Lambda, \Lambda^{\prime}\right)$ to another bimodule by using a homomorphism $\mathcal{C}_{\Lambda} \rightarrow S$ (where $S$ is a unital ring), we will necessarily obtain a free bimodule over a ring which does not satisfy the rank property. 


\section{Proof of Proposition 1.12}

We start by showing that there must exist at least three Reeb chords on a horizontally displaceable Legendrian submanifold whose Chekanov-Eliashberg algebra is not acyclic, but whose characteristic algebra admits no finite-dimensional representations. In particular, we prove that the bound $|\mathcal{Q}(\Lambda)|<3$ contradicts the hypothesis.

In the case when $|\mathcal{Q}(\Lambda)|=1$, the differential must be trivial, and hence the characteristic algebra is free and obviously has a finite-dimensional representation.

In the case when $|\mathcal{Q}(\Lambda)|=2$, the characteristic algebra is the quotient of the ChekanovEliashberg algebra $\langle a, b\rangle, \ell(a) \leq \ell(b)$, by the two-sided ideal generated by a polynomial $p(a)$ (which is not a unit, by assumption). In other words, the characteristic algebra is the free product $\mathbb{F}[b] * \mathbb{F}[a] /\langle p(a)\rangle$, which, hence, admits a unital projection

$$
\mathbb{F}[b] * \mathbb{F}[a] /\langle p(a)\rangle \rightarrow \mathbb{F}[b] * \mathbb{F}[a] /\langle p(a), b\rangle=\mathbb{F}[a] /\langle p(a)\rangle
$$

to a non-zero commutative unital algebra. Finally, observe that $\mathbb{F}[a] /\langle p(a)\rangle$ has a 1dimensional representation $\mathbb{F}[a] /\langle p(a)\rangle \rightarrow(\mathbb{F}[a] /\langle p(a)\rangle) / I$, where $I$ is any maximal ideal of the commutative ring $\mathbb{F}[a] /\langle p(a)\rangle$.

Remark 6.1 At least algebraically, there are examples of free DGAs with three generators for which the homology is not acyclic but for which the characteristic algebra admits no finite-dimensional representations. Consider the DGA generated by $\langle a, b, c\rangle$ over a field of characteristic zero for which $\partial(a)=\partial(b)=0$ and $\partial(c)=1-(a b-b a)$. A finite-dimensional unital representation of the characteristic algebra must send $a b-b a$ to the identity. Since the trace of a commutator vanishes, while the trace of the identity is non-zero, such a representation cannot exist.

We now prove Proposition 1.12 in case (1). Let $c_{\text {even }}$ and $c_{\text {odd }}$ denote the number of generators in even and odd degree, respectively. Using [8, Proposition 3.3] we can express the Thurston-Bennequin number as

$$
\operatorname{tb}(\Lambda)=(-1)^{(n-2)(n-1) / 2}\left(c_{\text {even }}-c_{\text {odd }}\right)=(-1)^{k+1}\left(c_{\text {even }}-c_{\text {odd }}\right) .
$$

Combining this with the identity

$$
\operatorname{tb}(\Lambda)=(-1)^{k+1} \frac{1}{2} \chi(\Lambda)
$$

from [8, Proposition 3.2(2)], we conclude that

$$
c_{\text {even }}=\frac{1}{2}|\chi(\Lambda)|+c_{\text {odd }}
$$


holds under the additional assumption that $\chi(\Lambda) \geq 0$. Moreover, the equality $c_{\text {odd }}=0$ implies that the differential is trivial and, hence, that there is a canonical (graded) augmentation. We must therefore have $c_{\text {odd }} \geq 1$, from which it follows that

$$
|\mathcal{Q}(\Lambda)|=c_{\text {odd }}+c_{\text {even }}=\frac{1}{2}|\chi(\Lambda)|+2 c_{\text {odd }} \geq \frac{1}{2}|\chi(\Lambda)|+2 .
$$

The proof in case (2) is analogous. It suffices to consider the case $\chi(\Lambda)<0$, for which the above expressions yield

$$
c_{\text {odd }}=\frac{1}{2}|\chi(\Lambda)|+c_{\text {even }} .
$$

The assumption that the Maslov class is non-vanishing, ie that the Chekanov-Eliashberg algebra admits an integer-valued grading, together with the assumption that all generators are of non-negative degree, has the following strong implication. Given that $c_{\text {even }}=0$, the Chekanov-Eliashberg algebra actually vanishes in degree zero, from which the existence of an augmentation follows (using the assumption that the DGA is not acyclic). We must thus have $c_{\text {even }} \geq 1$, from which we obtain the sought inequality

$$
|\mathcal{Q}(\Lambda)|=c_{\text {odd }}+c_{\text {even }}=\frac{1}{2}|\chi(\Lambda)|+2 c_{\text {even }} \geq \frac{1}{2}|\chi(\Lambda)|+2 .
$$

\section{References}

[1] V I Arnol'd, The first steps of symplectic topology, Uspekhi Mat. Nauk 41 (1986) 3-18, 229 MR890489 In Russian; translated in Russian Math. Surv. 41 (1986) 1-21

[2] M Audin, Fibrés normaux d'immersions en dimension double, points doubles d'immersions lagragiennes et plongements totalement réels, Comment. Math. Helv. 63 (1988) 593-623 MR966952

[3] D Bennequin, Entrelacements et équations de Pfaff, from: "Third Schnepfenried geometry conference, I", Astérisque 107, Soc. Math. France, Paris (1983) 87-161 MR753131

[4] Y Chekanov, Differential algebra of Legendrian links, Invent. Math. 150 (2002) 441483 MR1946550

[5] G Dimitroglou Rizell, Legendrian ambient surgery and Legendrian contact homology, preprint (2014) arXiv: 1205.5544 to appear in J. Sympl. Top.

[6] T Ekholm, Y Eliashberg, E Murphy, I Smith, Constructing exact Lagrangian immersions with few double points, Geom. Funct. Anal. 23 (2013) 1772-1803 MR3132903

[7] T Ekholm, J B Etnyre, J M Sabloff, A duality exact sequence for Legendrian contact homology, Duke Math. J. 150 (2009) 1-75 MR2560107

[8] T Ekholm, J Etnyre, M Sullivan, Non-isotopic Legendrian submanifolds in $\mathbb{R}^{2 n+1}$, J. Differential Geom. 71 (2005) 85-128 MR2191769 
[9] T Ekholm, J Etnyre, M Sullivan, Orientations in Legendrian contact homology and exact Lagrangian immersions, Internat. J. Math. 16 (2005) 453-532 MR2141318

[10] T Ekholm, J Etnyre, M Sullivan, Legendrian contact homology in $P \times \mathbb{R}$, Trans. Amer. Math. Soc. 359 (2007) 3301-3335 MR2299457

[11] T Ekholm, K Honda, T Kálmán, Legendrian knots and exact Lagrangian cobordisms, preprint (2012) arXiv:1212.1519 to appear in J. Eur. Math. Soc.

[12] T Ekholm, T Kálmán, Isotopies of Legendrian 1-knots and Legendrian 2-tori, J. Symplectic Geom. 6 (2008) 407-460 MR2471099

[13] Y Eliashberg, A Givental, H Hofer, Introduction to symplectic field theory, Geom. Funct. Anal. (2000) 560-673 MR1826267

[14] Y Eliashberg, E Murphy, Lagrangian caps, Geom. Funct. Anal. 23 (2013) 1483-1514 MR3102911

[15] J Epstein, D Fuchs, On the invariants of Legendrian mirror torus links, from: "Symplectic and contact topology: interactions and perspectives", (Y Eliashberg, B Khesin, F Lalonde, editors), Fields Inst. Commun. 35, Amer. Math. Soc. (2003) 103-115 MR1969270

[16] J B Etnyre, K Honda, On connected sums and Legendrian knots, Adv. Math. 179 (2003) 59-74 MR2004728

[17] R Golovko, A note on the front spinning construction, Bull. Lond. Math. Soc. 46 (2014) 258-268 MR3194745

[18] M Gromov, Pseudoholomorphic curves in symplectic manifolds, Invent. Math. 82 (1985) 307-347 MR809718

[19] M B Henry, D Rutherford, Ruling polynomials and augmentations over finite fields, J. Topol. 8 (2015) 1-37 MR3335247

[20] K H Ko, S Lee, On Kauffman polynomials of links, J. Korean Math. Soc. 26 (1989) 33-42 MR1005865

[21] P Lambert-Cole, Legendrian products, preprint (2013) arXiv:1301.3700

[22] C Leverson, Augmentations and rulings of Legendrian knots, preprint (2014) arXiv: 1403.4982

[23] E Murphy, Loose Legendrian embeddings in high dimensional contact manifolds, preprint (2013) arXiv:1201.2245

[24] L L Ng, Computable Legendrian invariants, Topology 42 (2003) 55-82 MR1928645

[25] L Ng, D Rutherford, Satellites of Legendrian knots and representations of the Chekanov-Eliashberg algebra, Algebr. Geom. Topol. 13 (2013) 3047-3097 MR3116313

[26] L Rudolph, A congruence between link polynomials, Math. Proc. Cambridge Philos. Soc. 107 (1990) 319-327 MR1027784 
[27] D Rutherford, Thurston-Bennequin number, Kauffman polynomial, and ruling invariants of a Legendrian link: The Fuchs conjecture and beyond, Int. Math. Res. Not. 2006 (2006) MR2219227

[28] D Sauvaget, Curiosités lagrangiennes en dimension 4, Ann. Inst. Fourier (Grenoble) 54 (2004) 1997-2020 MR2134231

[29] S Sivek, The contact homology of Legendrian knots with maximal Thurston-Bennequin invariant, J. Symplectic Geom. 11 (2013) 167-178 MR3046489

[30] S L Tabachnikov, An invariant of a submanifold transversal to a distribution, Uspekhi Mat. Nauk 43 (1988) 193-194 MR955791 In Russian; translated in Russian Math. Surv. 43 (1988) 225-226

Department of Pure Mathematics and Mathematical Statistics Centre for Mathematical Sciences, University of Cambridge Wilberforce Road, Cambridge CB3 OWB, UK

Alfréd Rényi Institute of Mathematics, Hungarian Academy of Sciences Realtanoda u. 13-15, Budapest, H-1053, Hungary

g.dimitroglou@maths.cam.ac.uk, golovko.roman@renyi.mta.hu http://www.dimitroglou.name/, http://sites.google.com/site/ragolovko/

Received: 29 September 2014 Revised: 6 February 2015 